WUCG-21-13

\title{
Relativistic star perturbations in Horndeski theories with a gauge-ready formulation
}

\author{
Ryotaro Kase ${ }^{1}$ and Shinji Tsujikawa ${ }^{2}$ \\ ${ }^{1}$ Department of Physics, Faculty of Science, Tokyo University of Science, \\ 1-3, Kagurazaka, Shinjuku-ku, Tokyo 162-8601, Japan \\ ${ }^{2}$ Department of Physics, Waseda University, 3-4-1 Okubo, Shinjuku, Tokyo 169-8555, Japan
}

(Dated: January 28, 2022)

\begin{abstract}
We present a general framework for studying the relativistic star perturbations on a static and spherically symmetric background in full Horndeski theories. We take a perfect fluid into account as a form of the Schutz-Sorkin action. Our formulation is sufficiently versatile in that the second-order actions of perturbations in odd- and even-parity sectors are derived without choosing particular gauge conditions, so they can be used for any convenient gauges at hand. The odd-parity sector contains one dynamical gravitational degree of freedom coupled to a time-independent four velocity of the fluid. In the even-parity sector there are three dynamical perturbations associated with gravity, scalar field, and matter sectors, whose equations of motion are decoupled from other nondynamical perturbations. For high radial and angular momentum modes, we obtain the propagation speeds of all dynamical perturbations and show that the perfect fluid in the even-parity sector has a standard sound speed affected by neither gravity nor the scalar field. Our general stability conditions and perturbation equations of motion can be directly applied to the stabilities of neutron stars and black holes as well as the calculations of their quasi-normal frequencies.
\end{abstract}

\section{INTRODUCTION}

The physics of black holes (BHs) and neutron stars (NSs) can be probed by observing their binary systems through gravitational waves (GWs) $[1,2]$. Precise measurements of the GW waveform of a compact binary coalescence event yield a plethora of information about the accuracy of General Relativity (GR) and their possible deviations on a strong gravitational background [3-10]. Moreover the tidal deformation of a NS-NS binary system provides useful constraints on their mass-radius relation [11-13], so that the NS equations of state are probed by GW measurements $[14,15]$. After the merging of binaries the BHs and NSs exhibit quasi-normal oscillations [16-18], whose frequencies can be computed by studying perturbations in the odd- and even-parity sectors.

GR is a fundamental theory of gravity whose accuracy has been confirmed on the weak gravitational background [19]. However, it is yet unknown how much extent GR accurately describes the physics around strong gravitational objects. On the cosmological side, there are several unsolved problems like the origin of dark energy, dark matter, inflation, and the matter-antimatter asymmetry. The existence of such problems may imply the presence of extra degrees of freedom (DOFs) beyond those appearing in GR and standard model of particle physics [20-24]. Those extra DOFs can be a scalar field, vector field, or a massive graviton, and so on. Among them, the scalar field can be naturally accommodated on an isotropic and homogenous cosmological background, so it has been extensively applied to the physics of inflation and dark energy.

The theories in which a scalar field $\phi$ has nonminimal or derivative couplings to gravity are generally called scalartensor theories [25]. In particular, Horndeski theories [26] are the most general scalar-tensor theories with second-order field equations of motion (see also Refs. [27-29]). In subclasses of Horndeski theories, there have been many works for the search of "hairy" BH and NS solutions on a static and spherically symmetric background. In GR with a minimally coupled scalar field, there is a no-hair BH theorem stating that the BHs are described by only three parameters, i.e., mass, electric charge, and angular momentum [30,31]. This property also holds for a nonminimally coupled scalar field with the Ricci scalar $R$ [32-34] (e.g., Brans-Dicke theories [35]). In the presence of a Gauss-Bonnet term linearly coupled to $\phi$, there exists a hairy BH solution where the background geometry is affected by the scalar field [36, 37]. If we do not impose the asymptotic flatness, nonminimal derivative couplings to the Einstein tensor $G_{\mu \nu}$ give rise to hairy BH solutions with a nonvanishing scalar-field profile $\phi(r)$ [38-40]. It is also known that a time-dependent scalar field of the form $\phi=q t+\psi(r)$ allows the existence of a nontrivial stealth Schwarzschild solution [41-45].

In scalar-tensor theories with a nonminimal coupling of the form $F(\phi) R$, there exist some static and spherically symmetric NS solutions with scalar hairs [46-55]. For the coupling $F(\phi)$ containing even power-law functions of $\phi$, there is a nontrivial hairy branch $(\phi \neq 0)$ besides the standard general relativistic branch $(\phi=0$ everywhere). If the second derivative $\mathrm{d}^{2} F / \mathrm{d} \phi^{2}$ is positive at $\phi=0$, the GR branch can be unstable to trigger tachyonic growth of $\phi$ toward the hairy branch. This phenomenon, which is induced by a tachyonic mass of the scalar field at $\phi=0$, is dubbed spontaneous scalarization [46, 47]. In the presence of a Gauss-Bonnet term coupled to the scalar field, it has been recognized that spontaneous scalarization can also occur for nonrotating and rotating BHs [56-61].

Nonminimally coupled theories with the coupling $F(\phi) R$ include Brans-Dicke theories with a scalar potential and $f(R)$ gravity. In these theories the nonminimal coupling $F(\phi)$ is not given by even power-law functions of $\phi$, so 
spontaneous scalarization does not occur. Applying the $f(R)$ models of late-time cosmic acceleration to relativistic stars [62-65], the existence of a growing scalar mass in regions of high density can give rise to a rapidly increasing solution of the scalar degree of freedom. In this case, the fine tuning of boundary conditions is required at the center of spherical symmetry. NS solutions in Starobinsky model [66], which is described by the Lagrangian $f(R)=$ $R+R^{2} /\left(6 m^{2}\right)$ with a constant mass $m$, were studied in Ref. [53] by using a non-perturbative approach both inside and outside the NS. (see Refs. [48, 49] for a perturbative approach). There are also models with the Lagrangian $f(R)=R+a R^{p}$, where $p$ is a constant in the range $1<p<2$. In this case, the effective scalar mass depends on the field value and approaches 0 toward spatial infinity. NS solutions of this class of models and its cooling process were studied in Refs. [55, 67]. In a subclass of derivative coupling theories where the scalar field is coupled to an Einstein tensor $G_{\mu \nu}$ of the form $\phi G_{\mu \nu} \nabla^{\mu} \nabla^{\nu} \phi$, there are also nontrivial NS solutions endowed with a scalar hair only inside the star $[68-70]$.

To study the stabilities of hairy BHs and NSs in scalar-tensor theories, we need to consider the perturbations on a static and spherically symmetric background. For BHs, this issue was addressed in full Horndeski theories for odd-parity modes [71] and even-parity modes [72] (see Refs. [73-78] for the application to quasinormal modes). For NSs, the analysis of perturbations with matter was carried out in Ref. [79] in a subclass of Horndeski theories given by the Lagrangian $\mathcal{L}=G_{2}(\phi, X)+G_{4}(\phi) R$. The stability of derivative coupling theories containing the Lagrangian $\phi G_{\mu \nu} \nabla^{\mu} \nabla^{\nu} \phi$ was also performed in Ref. [80]. However, the general stability conditions of NSs in full Horndeski theories have not been derived yet.

The Lagrangian of full Horndeski theories contains the cubic self-interaction $G_{3}(\phi, X) \square \phi$ as well as nonminimal and derivatives couplings to gravity, see Eq. (2.2) in Sec. II. On the expanding cosmological background, the Lagrangian of theories with the speed of gravitational waves $c_{t}$ exactly equivalent to that of light $(c)$ is constrained to be of the form $\mathcal{L}=G_{2}(\phi, X)+G_{3}(\phi, X) \square \phi+G_{4}(\phi) R$. From the gravitational wave event GW170817 [2] together with the $\gamma$-ray observation of its electromagnetic counterpart [81], there is a constraint $-3 \times 10^{-15} \leq c_{t}-1 \leq 7 \times 10^{-16}$ in the unit of $c=1$. The nonminimal derivative coupling $G_{5}(\phi) \propto \phi$ and linear Gauss-Bonnet coupling $G_{5}(X) \propto \ln X$ lead to the deviation of $c_{t}$ from 1 . However, the deviation usually occurs only in the vicinity of strong gravitational objects, so the $\mathrm{BH}$ and NS solutions in Horndeski theories containing the couplings $G_{4}(X)$ and $G_{5}(\phi, X)$ are not necessarily excluded from the observational bound of speed of gravitational waves.

In this paper, we will compute the second-order actions of odd- and even-parity perturbations in full Horndeski theories in the presence of a perfect fluid described by the Schutz-Sorkin action. We do not fix gauge conditions from the beginning, so the resulting perturbation equations of motion can be applied to any gauges. We will derive stability conditions of relativistic stars against odd- and even-parity perturbations for high radial and angular momentum modes. For BHs, i.e., in the absence of the matter fluid, the similar investigations were performed in Refs. [71, 72], but the stability of even-parity perturbations along the angular direction was not addressed. In the presence of matter, we will obtain all the stability conditions including those in the angular direction. Thus, our analysis is sufficiently general to accommodate the stabilities of NSs as well as BHs.

For the integration of the matter action, the prescriptions taken in Refs. [79, 80] contain some inappropriate points. In this paper, we will also correct them and show that the dynamical matter perturbation in the even-parity sector propagates with the standard sound speed $c_{m}$ affected by neither gravity nor the scalar field. We will also apply our results to derivative coupling theories containing the Lagrangian $\phi G_{\mu \nu} \nabla^{\mu} \nabla^{\nu} \phi$ and show that the main results of instabilities of hairy solutions in this class of theories [80] are not subject to modifications. We would like to stress that our general gauge-ready formulation of relativistic star perturbations in full Horndeski theories can be directly applied to the computations of quasi-normal modes of NSs and BHs as well as to the tidal deformation of NSs.

\section{HORNDESKI THEORIES WITH MATTER AND BACKGROUND EQUATIONS ON A STATIC AND SPHERICALLY SYMMETRIC BACKGROUND}

The action of most general scalar-tensor theories with second-order field equations of motion is given by [26-28]

$$
\mathcal{S}=\int \mathrm{d}^{4} x \sqrt{-g} \mathcal{L}_{H}+\mathcal{S}_{m}\left(g_{\mu \nu}, \Psi_{m}\right),
$$

where $g$ is a determinant of the metric tensor $g_{\mu \nu}$, and

$$
\begin{aligned}
\mathcal{L}_{H}= & G_{2}(\phi, X)-G_{3}(\phi, X) \square \phi+G_{4}(\phi, X) R+G_{4, X}(\phi, X)\left[(\square \phi)^{2}-\left(\nabla_{\mu} \nabla_{\nu} \phi\right)\left(\nabla^{\mu} \nabla^{\nu} \phi\right)\right]+G_{5}(\phi, X) G_{\mu \nu} \nabla^{\mu} \nabla^{\nu} \phi \\
& -\frac{1}{6} G_{5, X}(\phi, X)\left[(\square \phi)^{3}-3(\square \phi)\left(\nabla_{\mu} \nabla_{\nu} \phi\right)\left(\nabla^{\mu} \nabla^{\nu} \phi\right)+2\left(\nabla^{\mu} \nabla_{\alpha} \phi\right)\left(\nabla^{\alpha} \nabla_{\beta} \phi\right)\left(\nabla^{\beta} \nabla_{\mu} \phi\right)\right],
\end{aligned}
$$

with $R$ and $G_{\mu \nu}$ being the Ricci scalar and Einstein tensor, respectively. The four functions $G_{j}$ 's $(j=2,3,4,5)$ depend on the scalar field $\phi$ and its kinetic term $X=-g^{\mu \nu} \nabla_{\mu} \phi \nabla_{\nu} \phi / 2$, with the covariant derivative operator $\nabla_{\mu}$. We also 
use the notations $\square \phi \equiv \nabla^{\mu} \nabla_{\mu} \phi$ and $G_{j, \phi} \equiv \partial G_{j} / \partial \phi, G_{j, X} \equiv \partial G_{j} / \partial X, G_{j, \phi X} \equiv \partial^{2} G_{j} /(\partial X \partial \phi)$, etc.

For the matter fields $\Psi_{m}$ inside relativistic stars, we consider a perfect fluid minimally coupled to gravity. This is described by the Schutz-Sorkin action [82-85]

$$
\mathcal{S}_{m}=-\int \mathrm{d}^{4} x\left[\sqrt{-g} \rho(n)+J^{\mu}\left(\partial_{\mu} \ell+\mathcal{A}_{i} \partial_{\mu} \mathcal{B}^{i}\right)\right]
$$

where $\rho$ is a matter density depending on its number density $n$, and $J^{\mu}$ is a vector field related to the fluid four velocity $u^{\mu}$ as

$$
u^{\mu}=\frac{J^{\mu}}{n \sqrt{-g}} .
$$

Due to the relation $u^{\mu} u_{\mu}=-1$, the fluid number density $n$ is expressed as

$$
n=\sqrt{\frac{g_{\mu \nu} J^{\mu} J^{\nu}}{g}} .
$$

The scalar field $\ell$ and spatial vectors $\mathcal{A}_{i}, \mathcal{B}^{i}(i=1,2,3)$ are the Lagrange multipliers arising in accompany with the current vector field $J^{\mu}$. In the matter action (2.3), we do not take an entropy mode into account.

\section{A. Variation of the matter action}

The covariant field equations of motion following from the Horndeski Lagrangian (2.2) are explicitly given in Ref. [28], so we do not repeat to write them here. In the following, we briefly revisit the field equations in the perfect-fluid sector. Varying the Schutz-Sorkin action (2.3) with respect to $J^{\mu}, \ell, \mathcal{A}_{i}$, and $\mathcal{B}^{i}$, respectively, we obtain

$$
\begin{aligned}
\partial_{\mu} \ell & =\rho_{, n} u_{\mu}-\mathcal{A}_{i} \partial_{\mu} \mathcal{B}^{i}, \\
\partial_{\mu} J^{\mu} & =0, \\
J^{\mu} \partial_{\mu} \mathcal{B}^{i} & =0, \\
J^{\mu} \partial_{\mu} \mathcal{A}_{i} & =0,
\end{aligned}
$$

where $\rho_{, n} \equiv \partial \rho / \partial n$. For the derivation of Eq. (2.9), we used the relation (2.7). Substituting $J^{\mu}=n \sqrt{-g} u^{\mu}$ into Eq. (2.7), it follows that

$$
u^{\mu} \nabla_{\mu} \rho+(\rho+P) \nabla_{\mu} u^{\mu}=0
$$

where $P$ is the matter pressure defined by

$$
P \equiv n \rho_{, n}-\rho
$$

The Lagrange multipliers $\ell, \mathcal{A}_{i}$, and $\mathcal{B}^{i}$ are related to the physical quantities $\rho$ and $u^{\mu}$ through Eqs. (2.6), (2.8), and (2.9). We will see that these Lagrange multipliers finally disappear from the background and perturbation equations of motion.

As shown in Refs. [79, 86], varying the matter Lagrangian $L_{m}=-\left[\sqrt{-g} \rho(n)+J^{\mu}\left(\partial_{\mu} \ell+\mathcal{A}_{i} \partial_{\mu} \mathcal{B}^{i}\right)\right]$ with respect to $g^{\mu \nu}$ leads to a familiar form of the perfect-fluid energy momentum tensor

$$
T_{\mu \nu} \equiv-\frac{2}{\sqrt{-g}} \frac{\delta L_{m}}{\delta g^{\mu \nu}}=(\rho+P) u_{\mu} u_{\nu}+P g_{\mu \nu},
$$

where we used the relation (2.6). Since the matter sector is minimally coupled to gravity, it obeys the continuity equation

$$
\nabla^{\mu} T_{\mu \nu}=0
$$

Multiplying $u^{\nu}$ with Eq. (2.13), the resulting equation is identical to Eq. (2.10). Introducing a unit vector $n^{\nu}$ orthogonal to $u_{\nu}$ (i.e., $n^{\nu} u_{\nu}=0$ and $n^{\nu} n_{\nu}=1$ ) and multiplying $n^{\nu}$ with Eq. (2.13), we obtain

$$
n_{\mu} \nabla^{\mu} P=-(\rho+P) n^{\nu} u_{\mu} \nabla^{\mu} u_{\nu}
$$

If we apply this relation to the configuration of compact stars, it can be interpreted as a balance between the pressure gradient and gravity [79, 87]. 


\section{B. Background equations}

We consider a static and spherically symmetric background given by the line element

$$
\mathrm{d} s^{2}=-f(r) \mathrm{d} t^{2}+h^{-1}(r) \mathrm{d} r^{2}+r^{2}\left(\mathrm{~d} \theta^{2}+\sin ^{2} \theta \mathrm{d} \varphi^{2}\right)
$$

where $f(r)$ and $h(r)$ are functions of the distance $r$ from the center of symmetry. On this background, we consider the radial dependent scalar field,

$$
\phi=\phi(r)
$$

For the matter sector, we can take the following configurations,

$$
J^{\mu}=[\sqrt{-g} N(r), 0,0,0], \quad \mathcal{A}_{i}=0
$$

where $\sqrt{-g}=f^{1 / 2} h^{-1 / 2} r^{2} \sin \theta$. From Eq. (2.5), the quantity $N(r)$ is related to the fluid number density $n$, as

$$
n(r)=f(r)^{1 / 2} N(r)
$$

Substituting Eqs. (2.17) and (2.18) into Eq. (2.4), the fluid four velocity is expressed in the form

$$
u^{\mu}=\left[f(r)^{-1 / 2}, 0,0,0\right] .
$$

Varying the action (2.1) with respect to $g_{\mu \nu}$, the $(00),(11),(22)$ components of gravitational field equations of motion are given, respectively, by

$$
\begin{aligned}
\mathcal{E}_{00} \equiv & \left(A_{1}+\frac{A_{2}}{r}+\frac{A_{3}}{r^{2}}\right) \phi^{\prime \prime}+\left(\frac{\phi^{\prime}}{2 h} A_{1}+\frac{A_{4}}{r}+\frac{A_{5}}{r^{2}}\right) h^{\prime}+A_{6}+\frac{A_{7}}{r}+\frac{A_{8}}{r^{2}}=\rho, \\
\mathcal{E}_{11} \equiv & -\left(\frac{\phi^{\prime}}{2 h} A_{1}+\frac{A_{4}}{r}+\frac{A_{5}}{r^{2}}\right) \frac{h f^{\prime}}{f}+A_{9}-\frac{2 \phi^{\prime}}{r} A_{1}-\frac{1}{r^{2}}\left[\frac{\phi^{\prime}}{2 h} A_{2}+(h-1) A_{4}\right]=P, \\
\mathcal{E}_{22} \equiv & {\left[\left\{A_{2}+\frac{(2 h-1) \phi^{\prime} A_{3}+2 h A_{5}}{h \phi^{\prime} r}\right\} \frac{f^{\prime}}{4 f}+A_{1}+\frac{A_{2}}{2 r}\right] \phi^{\prime \prime}+\frac{1}{4 f}\left(2 h A_{4}-\phi^{\prime} A_{2}+\frac{2 h A_{5}-\phi^{\prime} A_{3}}{r}\right)\left(f^{\prime \prime}-\frac{f^{\prime 2}}{2 f}\right) } \\
& +\left[A_{4}+\frac{2 h(2 h+1) A_{5}-\phi^{\prime} A_{3}}{2 h^{2} r}\right] \frac{f^{\prime} h^{\prime}}{4 f}+\left(\frac{A_{7}}{4}+\frac{A_{10}}{r}\right) \frac{f^{\prime}}{f}+\left(\frac{\phi^{\prime}}{h} A_{1}+\frac{A_{4}}{r}\right) \frac{h^{\prime}}{2}+A_{6}+\frac{A_{7}}{2 r}=-P, \quad(2.22
\end{aligned}
$$

where a prime represents the derivative with respect to $r$. The coefficients are given by

$$
\begin{aligned}
& A_{1}=-h^{2}\left(G_{3, X}-2 G_{4, \phi X}\right) \phi^{2}-2 G_{4, \phi} h \\
& A_{2}=2 h^{3}\left(2 G_{4, X X}-G_{5, \phi X}\right) \phi^{\prime 3}-4 h^{2}\left(G_{4, X}-G_{5, \phi}\right) \phi^{\prime} \\
& A_{3}=-h^{4} G_{5, X X} \phi^{4}+h^{2} G_{5, X}(3 h-1) \phi^{\prime 2} \\
& A_{4}=h^{2}\left(2 G_{4, X X}-G_{5, \phi X}\right) \phi^{\prime 4}+h\left(3 G_{5, \phi}-4 G_{4, X}\right) \phi^{\prime 2}-2 G_{4} \\
& A_{5}=-\frac{1}{2}\left[G_{5, X X} h^{3} \phi^{\prime 5}-h G_{5, X}(5 h-1) \phi^{\prime 3}\right] \\
& A_{6}=h\left(G_{3, \phi}-2 G_{4, \phi \phi}\right) \phi^{\prime 2}+G_{2} \\
& A_{7}=-2 h^{2}\left(2 G_{4, \phi X}-G_{5, \phi \phi}\right) \phi^{\prime 3}-4 G_{4, \phi} h \phi^{\prime}, \\
& A_{8}=G_{5, \phi X} h^{3} \phi^{\prime 4}-h\left(2 G_{4, X} h-G_{5, \phi} h-G_{5, \phi}\right) \phi^{\prime 2}-2 G_{4}(h-1), \\
& A_{9}=-h\left(G_{2, X}-G_{3, \phi}\right) \phi^{\prime 2}-G_{2}, \\
& A_{10}=\frac{1}{2} G_{5, \phi X} h^{3} \phi^{\prime 4}-\frac{1}{2} h^{2}\left(2 G_{4, X}-G_{5, \phi}\right) \phi^{\prime 2}-G_{4} h .
\end{aligned}
$$

The matter continuity Eq. (2.14) translates to

$$
\mathcal{E}_{P} \equiv P^{\prime}+\frac{f^{\prime}}{2 f}(\rho+P)=0 .
$$


Variation of the action (2.1) with respect to $\phi$ leads to the scalar-field equation of motion

$$
\frac{1}{r^{2}} \sqrt{\frac{h}{f}}\left(r^{2} \sqrt{\frac{f}{h}} J^{r}\right)^{\prime}+\frac{\partial \mathcal{E}}{\partial \phi}=0,
$$

where

$$
\begin{aligned}
J^{r}= & \left(A_{1}+\frac{A_{2}}{r}+\frac{A_{3}}{r^{2}}\right) \frac{f^{\prime}}{2 f}-\frac{A_{6}+A_{9}}{\phi^{\prime}}+\frac{2}{r} A_{1}+\frac{1}{r^{2}}\left(\frac{1+h}{2 h} A_{2}-\frac{A_{4}+A_{8}-2 A_{10}}{\phi^{\prime}}\right), \\
\mathcal{E}= & {\left[A_{1}+\frac{1}{r^{2}}\left(\frac{A_{3}}{2 h}-\frac{A_{5}}{\phi^{\prime}}\right)\right]\left(\phi^{\prime \prime}+\frac{\phi^{\prime} h^{\prime}}{2 h}\right)+\left[\frac{\phi^{\prime}}{2} A_{2}-h A_{4}+\frac{1}{2 r}\left(\frac{\phi^{\prime}}{2} A_{3}-h A_{5}\right)\right] \frac{f^{\prime}}{r f} } \\
& +A_{6}+\frac{1}{r^{2}}\left(\frac{\phi^{\prime}}{2} A_{2}-h A_{4}+A_{8}-2 A_{10}\right) .
\end{aligned}
$$

In shift-symmetric Horndeski theories where the couplings $G_{j}(j=2,3,4,5)$ contain the $X$ dependence alone, we have $\partial \mathcal{E} / \partial \phi=0$ in Eq. (2.25). In this case, the scalar-field equation reduces to $J^{r}=\left(Q / r^{2}\right) \sqrt{h / f}$, where $Q$ is a constant.

Combining Eqs. (2.20)-(2.22), and (2.24), the scalar-field equation (2.25) can be also expressed in the form

$$
\mathcal{E}_{\phi} \equiv-\frac{2}{\phi^{\prime}}\left[\frac{f^{\prime}}{2 f} \mathcal{E}_{00}+\mathcal{E}_{11}^{\prime}+\left(\frac{f^{\prime}}{2 f}+\frac{2}{r}\right) \mathcal{E}_{11}+\frac{2}{r} \mathcal{E}_{22}+\mathcal{E}_{P}\right]=0
$$

For a given equation of state $P=P(\rho)$ and a boundary condition around the center of star, we can solve Eqs. (2.20), $(2.21),(2.24)$, and $(2.25)$ for $f, h, P$, and $\phi$.

\section{Decomposition of perturbations}

We consider metric perturbations $h_{\mu \nu}$ on top of the background metric $\bar{g}_{\mu \nu}$ besides perturbations of the scalar field and perfect fluid. In doing so, we classify them into the odd- and even-parity sectors depending on the types of parities under the rotation in two-dimensional plane $(\theta, \varphi)[88,89]$. We expand the perturbations in terms of the spherical harmonics $Y_{l m}(\theta, \varphi)$. Under the two-dimensional rotation, the odd- and even-modes have the parities $(-1)^{l+1}$ and $(-1)^{l}$, respectively. Any scalar perturbation has the even-mode alone, whereas vector and tensor perturbations contain both odd- and even-modes.

To decompose the perturbations into the odd- and even-parity sectors, we introduce a tensor field

$$
E_{a b}=\sqrt{\gamma} \varepsilon_{a b},
$$

where $\gamma$ is the determinant of two-dimensional metric given by the line element $\mathrm{d} s_{(2)}^{2}=\gamma_{a b} \mathrm{~d} x^{a} \mathrm{~d} x^{b}=\mathrm{d} \theta^{2}+\sin ^{2} \theta \mathrm{d} \varphi^{2}$ (with $a, b$ either $\theta, \varphi$ ) and $\varepsilon_{a b}$ is an anti-symmetric symbol with $\varepsilon_{\theta \varphi}=1$. According to this definition of $E_{a b}$, we have $E_{\theta \varphi}=-E_{\varphi \theta}=\sin \theta$. Any scalar perturbation $S$ as well as the $\theta, \varphi$ components of vector and tensor perturbations $V_{\mu}$ and $T_{\mu \nu}$ can be expressed, respectively, as

$$
\begin{aligned}
S & =\sum_{l, m} S_{1}(t, r) Y_{l m}(\theta, \varphi), \\
V_{a} & =\sum_{l, m} V_{1}(t, r) \nabla_{a} Y_{l m}(\theta, \varphi)+\sum_{l, m} V_{2}(t, r) E_{a b} \nabla^{b} Y_{l m}(\theta, \varphi), \\
T_{a b} & =\sum_{l, m}\left[T_{1}(t, r) g_{a b} Y_{l m}+T_{2}(t, r) \nabla_{a} \nabla_{b} Y_{l m}\right]+\frac{1}{2} \sum_{l, m} T_{3}(t, r)\left[E_{a}{ }^{c} \nabla_{c} \nabla_{b} Y_{l m}+E_{b}{ }^{c} \nabla_{c} \nabla_{a} Y_{l m}\right],
\end{aligned}
$$

where $S_{1}, V_{1}, V_{2}, T_{1}, T_{2}$, and $T_{3}$ are functions of $t$ and $r$ (for which we omit the subscripts " $l m$ "), and $\nabla_{a}$ is a covariant derivative operator defined on the two-dimensional sphere. The odd- and even-parity modes correspond to those containing the functions $V_{2}, T_{3}$ and $S_{1}, V_{1}, T_{1}, T_{2}$, respectively [90-92].

In Secs. III and IV, we first apply the decompositions (2.30)-(2.32) to the perturbations associated with fundamental fields $h_{\mu \nu}, \phi$, as well as the scalar and vector quantities, $\ell, J^{\mu}, \mathcal{A}_{i}$, and $\mathcal{B}_{i}$, appearing in the matter action (2.3). Then, we expand all the quantities composed of these fundamental fields in the action (2.1), e.g., Ricci scalar $R$, kinetic term $X$ of the scalar field, number density $n$, energy density $\rho$, up to second order in perturbations. The resulting quadratic-order actions of odd- and even-parity sectors will be used to derive the perturbation equations of motion and stability conditions. 


\section{ODD-PARITY PERTURBATIONS}

In this section, we study the propagation of odd-parity perturbations in Horndeski theories with the matter action given by $(2.3)$. Under a rotation in the $(\theta, \varphi)$ plane, the $h_{t t}, h_{t r}$, and $h_{r r}$ components of metric perturbations $h_{\mu \nu}$ transform as scalars. The $h_{t a}$ and $h_{r a}$ components behave as vectors (with $a, b$ either $\theta, \varphi$ ), while $h_{a b}$ transforms as a tensor. Then, from Eqs. (2.30)-(2.32), the metric components in the odd-parity sector are given by

$$
\begin{aligned}
h_{t t} & =h_{t r}=h_{r r}=0, \\
h_{t a} & =\sum_{l, m} Q(t, r) E_{a b} \nabla^{b} Y_{l m}(\theta, \varphi), \quad h_{r a}=\sum_{l, m} W(t, r) E_{a b} \nabla^{b} Y_{l m}(\theta, \varphi), \\
h_{a b} & =\frac{1}{2} \sum_{l, m} U(t, r)\left[E_{a}{ }^{c} \nabla_{c} \nabla_{b} Y_{l m}(\theta, \varphi)+E_{b}{ }^{c} \nabla_{c} \nabla_{a} Y_{l m}(\theta, \varphi)\right],
\end{aligned}
$$

where $Q, W$, and $U$ are functions of $t$ and $r$. The scalar perturbation in the odd-parity sector vanishes, so the field $\phi$ has the background value $\bar{\phi}(r)$ alone, i.e.,

$$
\phi=\bar{\phi}(r) .
$$

In the following, we omit a bar for brevity.

For the matter sector, the scalar quantities $\rho$ and $n$ appearing in the action (2.3) do not have odd-parity perturbations. The components of $J_{\mu}$ associated with the odd-parity sector are given by

$$
J_{t}=\bar{J}_{t}, \quad J_{r}=0, \quad J_{a}=\sum_{l, m} \sqrt{-\bar{g}} \delta j(t, r) E_{a b} \nabla^{b} Y_{l m}(\theta, \varphi),
$$

where $\bar{J}_{t}=-n(f / \sqrt{h}) r^{2} \sin \theta$ and $\sqrt{-\bar{g}}=\sqrt{f / h} r^{2} \sin \theta$ are the background values. From Eq. (2.4), the perturbation $\delta j$ is related to the $\theta, \varphi$ components of four velocity $u^{a}$. The spatial vectors $\mathcal{A}_{i}$ and $\mathcal{B}^{i}$, where $i=r, \theta, \varphi$, are expressed in the forms

$$
\begin{aligned}
& \mathcal{A}_{i}=\delta \mathcal{A}_{i}, \quad \text { with } \quad \delta \mathcal{A}_{r}=0, \quad \delta \mathcal{A}_{a}=\sum_{l, m} \delta \mathcal{A}(t, r) E_{a b} \nabla^{b} Y_{l m}(\theta, \varphi), \\
& \mathcal{B}^{i}=x^{i}+\delta \mathcal{B}^{i}, \quad \text { with } \quad \delta \mathcal{B}^{r}=0, \quad \delta \mathcal{B}^{a}=\sum_{l, m} \delta \mathcal{B}(t, r) E^{a}{ }_{b} \nabla^{b} Y_{l m}(\theta, \varphi) .
\end{aligned}
$$

The perturbations $\delta \mathcal{A}$ and $\delta \mathcal{B}$ are related to $\delta j$ according to Eqs. (2.8) and (2.9). From Eq. (2.6), the Lagrange multiplier $\ell$ in the odd-parity sector obeys $\partial_{t} \ell=\rho_{, n}(r) u_{t}$ with $u_{t}=-\sqrt{f(r)}$. Integrating this relation gives

$$
\ell=-\rho_{, n}(r) \sqrt{f(r)} t
$$

where the integration constant is set to 0 (which does not affect the discussion below).

In what follows, we derive the second-order action of odd-parity perturbations for the purpose of obtaining linear perturbation equations of motion and associated stability conditions. Due to the spherical symmetry of the background, we can restrict ourselves to axisymmetric modes of perturbations $(m=0)$ by the suitable rotation of nonaxisymmetric modes $(m \neq 0)[93]$. Hence we will set $m=0$ without loss of generality and multiply $2 \pi$ for the integral with respect to $\varphi$.

\section{A. Second-order action}

We expand the action (2.1) up to second order in odd-parity perturbations for the multipoles $l \geq 2$ without choosing particular gauge conditions. The perturbation $\delta \phi$ of the scalar field $\phi$ vanishes, but the kinetic energy $X$ acquires a second-order contribution $\delta X=-h^{2} \phi^{\prime 2} W^{2} Y_{l 0, \theta}^{2} /\left(2 r^{2}\right)$ besides its background value $\bar{X}=-h \phi^{\prime 2} / 2$. From Eq. (2.5), the perturbation of the fluid number density expanded up to second-order is given by

$$
\delta n=\frac{n}{2 r^{2}}\left(h W^{2}-\frac{2}{f} Q^{2}+\frac{2}{n \sqrt{f}} Q \delta j-\frac{\delta j^{2}}{n^{2}}+\frac{U^{2}}{4 r^{2}} \cot ^{2} \theta\right) Y_{l 0, \theta}^{2}-\frac{n U^{2}}{4 r^{4}}\left(Y_{l 0, \theta} \cot \theta-\frac{1}{2} Y_{l 0, \theta \theta}\right) Y_{l 0, \theta \theta},
$$

where $n$ is the background value. The matter density in (2.3) contains its background value $\rho(r)$ and the perturbation $\rho_{, n} \delta n=(\rho+P) \delta n / n$. We also exploit Eqs. (3.5)-(3.7) for the expansion of the Schutz-Sorkin action. 
After expanding the total action (2.1) up to second order in perturbations, there are $\theta$-dependent terms containing the products such as $Y_{l 0, \theta} Y_{l 0, \theta \theta \theta}, Y_{l 0, \theta} Y_{l 0, \theta \theta}$, etc. After the integrations by parts, the resulting action possesses the terms proportional to $Y_{l 0, \theta}^{2}$ and $Y_{l 0, \theta \theta}^{2}$. These $\theta$-dependent terms can be integrated out from the action by employing the following relations [94]

$$
\int_{0}^{2 \pi} \mathrm{d} \varphi \int_{0}^{\pi} \mathrm{d} \theta Y_{l 0, \theta}^{2} \sin \theta=L, \quad \int_{0}^{2 \pi} \mathrm{d} \varphi \int_{0}^{\pi} \mathrm{d} \theta\left(\frac{Y_{l 0, \theta}^{2}}{\sin \theta}+Y_{l 0, \theta \theta}^{2} \sin \theta\right)=L^{2},
$$

where

$$
L \equiv l(l+1) .
$$

After this procedure, we perform the integrations by parts with respect to $t$ and $r$, and exploit the background equations presented in Sec. II B. Then, the second-order action in the odd-parity sector yields

$$
\mathcal{S}_{\text {odd }}=\sum_{l} L \int \mathrm{d} t \mathrm{~d} r \mathcal{L}_{\text {odd }}
$$

where

$$
\begin{aligned}
\mathcal{L}_{\text {odd }}= & \frac{\sqrt{h}}{4 \sqrt{f}} \mathcal{H}\left(\dot{W}-Q^{\prime}+\frac{2 Q}{r}\right)^{2}+(L-2)\left(\frac{\mathcal{F} Q^{2}}{4 r^{2} \sqrt{f h}}-\frac{\sqrt{f h}}{4 r^{2}} \mathcal{G} W^{2}\right)+\mathcal{L}_{U} \\
& +\frac{\sqrt{f}(\rho+P)}{2 n^{2} \sqrt{h}} \delta j^{2}-\frac{n\left(r^{2} \dot{\mathcal{B}}-Q\right)+\sqrt{f} \delta j}{\sqrt{h}} \delta \mathcal{A},
\end{aligned}
$$

with a dot being the derivative with respect to $t$. The variables $\mathcal{H}, \mathcal{F}$, and $\mathcal{G}$ are defined by

$$
\begin{aligned}
\mathcal{H} & \equiv 2 G_{4}+2 h \phi^{\prime 2} G_{4, X}-h \phi^{2} G_{5, \phi}-\frac{h^{2} \phi^{\prime 3} G_{5, X}}{r}, \\
\mathcal{F} & \equiv 2 G_{4}+h \phi^{\prime 2} G_{5, \phi}-h \phi^{\prime 2}\left(\frac{1}{2} h^{\prime} \phi^{\prime}+h \phi^{\prime \prime}\right) G_{5, X}, \\
\mathcal{G} & \equiv 2 G_{4}+2 h \phi^{\prime 2} G_{4, X}-h \phi^{\prime 2}\left(G_{5, \phi}+\frac{f^{\prime} h \phi^{\prime} G_{5, X}}{2 f}\right) .
\end{aligned}
$$

The Lagrangian $\mathcal{L}_{U}$, which arises from the gravitational perturbation $U$, is given by

$$
\begin{gathered}
\mathcal{L}_{U}=\frac{L-2}{8 r^{2} \sqrt{f h}}\left[\left\{2 \mathcal{F} \dot{Q}-2 f h \mathcal{G} W^{\prime}-\left(f^{\prime} h \mathcal{G}+f h^{\prime} \mathcal{G}+2 f h \mathcal{G}^{\prime}\right) W\right\} U+\frac{1}{2} \mathcal{F} \dot{U}^{2}-\frac{1}{2} f h \mathcal{G} U^{\prime 2}\right. \\
\left.-\frac{1}{2 r^{2}}\left(r f^{\prime} h \mathcal{G}+r f h^{\prime} \mathcal{G}+2 r f h \mathcal{G}^{\prime}-2 f h \mathcal{G}\right) U^{2}\right] .
\end{gathered}
$$

In the absence of the perfect fluid with the particular gauge choice $U=0$, the action (3.12) coincides with that derived in Ref. [71].

\section{B. Perturbation equations}

The second-order action (3.12) is written in a gauge-ready form ${ }^{1}$, so we can derive the linear perturbation equations of motion for any gauge choices of interest. Under the gauge transformation $x_{\mu} \rightarrow x_{\mu}+\xi_{\mu}$, where $\xi_{t}=0, \xi_{r}=0$, and $\xi_{a}=\sum_{l} \Lambda(t, r) E_{a b} \nabla^{b} Y_{l m}(\theta, \varphi)$, the three gravitational perturbations $Q, W$, and $U$ transform, respectively, as

$$
Q \rightarrow Q+\dot{\Lambda}, \quad W \rightarrow W+\Lambda^{\prime}-\frac{2}{r} \Lambda, \quad U \rightarrow U+2 \Lambda .
$$

\footnotetext{
${ }^{1}$ On an isotropic and homogenous cosmological background, the second-order perturbed action of full Horndeski theories was also derived in a gauge-ready form in Ref. [23].
} 
A commonly used gauge-invariant variable is

$$
\chi \equiv \dot{W}-Q^{\prime}+\frac{2 Q}{r},
$$

which was first introduced by Cunningham et al. [95]. This corresponds to a dynamical perturbation arising from the gravity sector.

First of all, varying the Lagrangian (3.13) with respect to $U$ leads to

$$
\begin{aligned}
& r^{2}\left[2 \mathcal{F} \dot{Q}-2 f h \mathcal{G} W^{\prime}-\left(f^{\prime} h \mathcal{G}+f h^{\prime} \mathcal{G}+2 f h \mathcal{G}^{\prime}\right) W\right]-r^{2} \mathcal{F} \ddot{U}+r^{2} f h \mathcal{G} U^{\prime \prime}+r^{4} \sqrt{f h}\left(\frac{\sqrt{f h} \mathcal{G}}{r^{2}}\right)^{\prime} U^{\prime} \\
& -\left(r f^{\prime} h \mathcal{G}+r f h^{\prime} \mathcal{G}+2 r f h \mathcal{G}^{\prime}-2 f h \mathcal{G}\right) U=0
\end{aligned}
$$

In the following, we choose the Regge-Wheeler gauge [88]

$$
U=0
$$

under which the gauge transformation scalar $\Lambda$ in Eq. (3.18) is completely fixed. Then, Eq. (3.20) reduces to

$$
2 \mathcal{F} \dot{Q}-2 f h \mathcal{G} W^{\prime}-\left(f^{\prime} h \mathcal{G}+f h^{\prime} \mathcal{G}+2 f h \mathcal{G}^{\prime}\right) W=0
$$

As we will see below, this relation is consistent with the other perturbation equations of motion

To derive the perturbation equation of $\chi$, we first vary the Lagrangian (3.13) with respect to $W$ and express $\ddot{W}$ by using $\dot{\chi}$. This leads to

$$
W=-\frac{r^{2} \mathcal{H}}{(L-2) f \mathcal{G}} \dot{\chi}
$$

Taking the time derivative of this relation and replacing $\dot{W}$ with $\chi+Q^{\prime}-2 Q / r$, it follows that

$$
r^{2} \mathcal{H} \ddot{\chi}+(L-2) f \mathcal{G}\left(\chi+Q^{\prime}-\frac{2 Q}{r}\right)=0 .
$$

Varying (3.13) with respect to $Q$, we obtain

$$
Q=\frac{r}{2(L-2) f \mathcal{F}}\left[\left(r h f^{\prime}-r f h^{\prime}-4 f h\right) \mathcal{H} \chi-2 r f h\left(\mathcal{H}^{\prime} \chi+\mathcal{H} \chi^{\prime}\right)-4 r f^{3 / 2} n \delta \mathcal{A}\right] .
$$

We take the $r$ derivative of Eq. (3.25) and express $Q^{\prime}$ and $Q$ in terms of $\chi, \delta \mathcal{A}$, and their $r$ derivatives. Then, Eq. (3.24) gives

$$
\ddot{\chi}-f h \frac{\mathcal{G}}{\mathcal{F}} \chi^{\prime \prime}+\alpha_{1} \chi^{\prime}+\frac{f}{r^{2}} \frac{\mathcal{G}}{\mathcal{H}}\left(L-2+\alpha_{2}^{\prime}-\frac{2}{r} \alpha_{2}\right) \chi-\frac{\sqrt{f} \mathcal{G}}{\mathcal{F}^{2} \mathcal{H}}\left[\left(n f^{\prime} \mathcal{F}+2 n^{\prime} f \mathcal{F}-2 n f \mathcal{F}^{\prime}\right) \delta \mathcal{A}+2 n f \mathcal{F} \delta \mathcal{A}^{\prime}\right]=0
$$

where

$$
\begin{aligned}
\alpha_{1} & \equiv-\frac{\mathcal{G}}{2 r \mathcal{F}^{2} \mathcal{H}}\left[\left\{\left(4 f h+3 r f h^{\prime}-r f^{\prime} h\right) \mathcal{H}+4 r f h \mathcal{H}^{\prime}\right\} \mathcal{F}-2 r f h \mathcal{H} \mathcal{F}^{\prime}\right] \\
\alpha_{2} & \equiv-\frac{r^{2} h \mathcal{H}}{\mathcal{F}}\left(\frac{\mathcal{H}^{\prime}}{\mathcal{H}}-\frac{f^{\prime}}{2 f}+\frac{h^{\prime}}{2 h}+\frac{2}{r}\right)
\end{aligned}
$$

The gravitational perturbation $\chi$ is coupled to the perfect fluid through the perturbation $\delta \mathcal{A}$. Varying the Lagrangian (3.13) with respect to $\delta \mathcal{A}, \delta \mathcal{B}$, and $\delta j$, respectively, we obtain

$$
\begin{aligned}
\dot{\delta \mathcal{B}} & =\frac{1}{r^{2}}\left(Q-\frac{\sqrt{f}}{n} \delta j\right), \\
\dot{\delta \mathcal{A}} & =0 \\
\delta \mathcal{A} & =\frac{\rho+P}{n^{2}} \delta j .
\end{aligned}
$$


Taking the $t$ and $r$ derivatives of Eq. (3.25) and Eq. (3.23), respectively, and using Eq. (3.30), one can confirm that Eq. (3.22) is consistently satisfied. From Eq. (3.31), the Lagrange multiplier $\delta \mathcal{A}$ is related to the physical perturbation $\delta j$. As we see in Eqs. (2.4) and (3.5), $\delta j$ corresponds to components $u_{a}$ of the fluid four velocity. From Eq. (3.30), $\delta \mathcal{A}$ depends on $r$ alone, so that

$$
\dot{\delta j}=0 \text {. }
$$

Substituting Eq. (3.31) and its $r$ derivative into Eq. (3.26), we find

$$
\ddot{\chi}-f h \frac{\mathcal{G}}{\mathcal{F}} \chi^{\prime \prime}+\alpha_{1} \chi^{\prime}+\frac{f}{r^{2}} \frac{\mathcal{G}}{\mathcal{H}}\left(L-2+\alpha_{2}^{\prime}-\frac{2}{r} \alpha_{2}\right) \chi=\frac{2 f^{3 / 2}(\rho+P) \mathcal{G}}{n \mathcal{F}^{2} \mathcal{H}}\left(\mathcal{F} \delta j^{\prime}-\mathcal{F}^{\prime} \delta j\right),
$$

so that the field $\chi$ is coupled to the perturbation $\delta j$. Since $\delta j$ is nondynamical due to the relation (3.32), the gravitational perturbation $\chi$ is the only dynamical degree of freedom. We have thus shown that the dynamics of odd-parity perturbation is governed by Eq. (3.33) with the nondynamical perturbation satisfying Eq. (3.32). These results are consistent with those derived in GR with a perfect fluid, see Eqs. (II-12a) and (II-12b) in Ref. [95].

Assuming the solution of Eq. (3.33) in the form

$$
\chi=\chi_{0} e^{i(\omega t-k r-l \theta)},
$$

where $\chi_{0}, \omega, k, l$ are constants, we derive the dispersion relation and propagation speeds of $\chi$. In the limits of large $\omega$ and $k$, and $l \gg 1$, we can ignore the terms $\alpha_{1} \chi^{\prime}, \alpha_{2}^{\prime} \chi, \alpha_{2} \chi / r$, and the right hand-side of Eq. (3.33), and hence

$$
\omega^{2}-f h \frac{\mathcal{G}}{\mathcal{F}} k^{2}-l^{2} \frac{f}{r^{2}} \frac{\mathcal{G}}{\mathcal{H}}=0 .
$$

In terms of the proper time $\tau=\int \sqrt{f} \mathrm{~d} t$ and the rescaled radial coordinate $r_{*}=\int \mathrm{d} r / \sqrt{h}$, the radial propagation speed is $c_{r}=\mathrm{d} r_{*} / \mathrm{d} \tau=\hat{c}_{r} / \sqrt{f h}$, where $\hat{c}_{r}=\mathrm{d} r / \mathrm{d} t=\omega / k$. To derive $c_{r}$, we drop the third term in Eq. (3.35) and substitute $\omega=k \sqrt{f h} c_{r}$ into Eq. (3.35). This leads to

$$
c_{r}^{2}=\frac{\mathcal{G}}{\mathcal{F}} .
$$

Similarly, the angular propagation speed in proper time is $c_{\Omega}=r \mathrm{~d} \theta / \mathrm{d} \tau=\hat{c}_{\Omega} / \sqrt{f}$, where $\hat{c}_{\Omega}=r \mathrm{~d} \theta / \mathrm{d} t$ satisfies $\omega^{2}=\hat{c}_{\Omega}^{2} l^{2} / r^{2}$. Substituting $\omega^{2}=c_{\Omega}^{2} f l^{2} / r^{2}$ into Eq. (3.35) and dropping the second term in Eq. (3.35), we obtain

$$
c_{\Omega}^{2}=\frac{\mathcal{G}}{\mathcal{H}} .
$$

To avoid the Laplacian instabilities along the radial and angular directions, we require the two conditions $c_{r}^{2} \geq 0$ and $c_{\Omega}^{2} \geq 0$. These conditions coincide with those derived in Ref. [71] in the absence of matter.

In Ref. [79], the relation (3.29) is plugged into the action (3.13), after which the term proportional to $\delta \mathcal{A}$ vanishes from the action. Then, we are left with the matter action proportional to $\delta j^{2}$. Variation of this action with respect to $\delta j$ leads to $\delta j=0$, in which case all the matter perturbations in (3.13) vanish. However, this procedure is not correct. The perturbation $\delta \mathcal{A}$ appears in the action only as a linear form $f(\delta j, Q, \delta \mathcal{B}) \delta \mathcal{A}$ and hence the variation with respect to $\delta \mathcal{A}$ leads to $f=0$, i.e., Eq. (3.29). The incorrect procedure is to substitute $f=0$ into the Lagrangian (3.13), after which the product $\delta j \delta \mathcal{A}$ disappears. When we vary the original Lagrangian (3.13) with respect to $\delta j$, the presence of this product gives rise to the coupling between $\delta \mathcal{A}$ and $\delta j$, see Eq. (3.31). Indeed, we have shown that the perturbation $\chi$ is coupled to $\delta j$ through the relation (3.31). In Ref. [79], the perturbation $\chi$ was introduced as a Lagrange multiplier at the action level, so we also take this approach in Sec. III C to clarify the issue of the elimination of nondynamical variables from the action.

\section{Action approach}

For the gauge choice $U=0$, the second-order Lagrangian (3.13) is equivalent to

$$
\begin{aligned}
\mathcal{L}_{\text {odd }}= & \frac{\sqrt{h}}{4 \sqrt{f}} \mathcal{H}\left[2 \chi\left(\dot{W}-Q^{\prime}+\frac{2 Q}{r}\right)-\chi^{2}\right]+(L-2)\left(\frac{\mathcal{F} Q^{2}}{4 r^{2} \sqrt{f h}}-\frac{\sqrt{f h}}{4 r^{2}} \mathcal{G} W^{2}\right) \\
& +\frac{\sqrt{f}(\rho+P)}{2 n^{2} \sqrt{h}} \delta j^{2}-\frac{n\left(r^{2} \dot{\mathcal{B}}-Q\right)+\sqrt{f} \delta j}{\sqrt{h}} \delta \mathcal{A},
\end{aligned}
$$


whose variation with respect to $\chi$ gives $\chi=\dot{W}-Q^{\prime}+2 Q / r$. Varying (3.38) with respect to $W$ and $Q$, respectively, we obtain the same equations as Eqs. (3.23) and (3.25). The Lagrangian (3.38) contains the terms $W^{2}$ and $Q^{2}$ besides $\dot{W}, Q^{\prime}$ and $Q$ (i.e., they do not appear as linear terms), so we can substitute the relations (3.23) and (3.25) as well as their $t$ and $r$ derivatives into Eq. (3.38). After the integrations by parts, the action reduces to the form (3.12), with the Lagrangian

$$
\mathcal{L}_{\text {odd }}=K_{\chi} \dot{\chi}^{2}+G_{\chi} \chi^{\prime 2}+M_{\chi} \chi^{2}+\mathcal{L}_{m}
$$

where

$$
\begin{aligned}
K_{\chi}= & \frac{r^{2} \sqrt{h} \mathcal{H}^{2}}{4(L-2) f^{3 / 2} \mathcal{G}}, \quad G_{\chi}=-f h \frac{\mathcal{G}}{\mathcal{F}} K_{\chi}, \quad M_{\chi}=-K_{\chi} \frac{f}{r^{2}} \frac{\mathcal{G}}{\mathcal{H}}\left(L-2+\alpha_{2}^{\prime}-\frac{2}{r} \alpha_{2}\right), \\
\mathcal{L}_{m}= & \frac{1}{2(L-2) f^{3 / 2} \sqrt{h} n^{2} \mathcal{F}}\left[(L-2)(\rho+P) f^{2} \mathcal{F} \delta j^{2}-2(L-2) f^{2} n^{2} \mathcal{F} \delta j \delta \mathcal{A}-2 r^{2} f^{2} n^{4} \delta \mathcal{A}^{2}\right. \\
& \left.-\left\{\left(2 r(L-2) \mathcal{F} \delta \dot{\mathcal{B}}+r\left(h^{\prime} \mathcal{H}+2 h \mathcal{H}^{\prime}\right) \chi+2 r h \mathcal{H} \chi^{\prime}+4 h \mathcal{H} \chi\right) r f^{3 / 2} n-\sqrt{f} f^{\prime} h n r^{2} \mathcal{H} \chi\right\} n^{2} \delta \mathcal{A}\right] .
\end{aligned}
$$

The matter Lagrangian $\mathcal{L}_{m}$ contains terms $\delta j^{2}$ and $\delta j \delta \mathcal{A}$, so the variation with respect to $\delta j$ gives $\delta \mathcal{A}=(\rho+P) \delta j / n^{2}$, i.e., Eq. (3.31). Substituting this relation into Eq. (3.41) to eliminate the Lagrange multiplier $\delta \mathcal{A}$, we obtain the reduced matter Lagrangian

$$
\begin{aligned}
\mathcal{L}_{m}= & -\frac{r(\rho+P)}{n \sqrt{h}}\left[r \delta j \delta \dot{\mathcal{B}}+\frac{\sqrt{f}\left\{(L-2) \mathcal{F}+2 r^{2}(\rho+P)\right\}}{2(L-2) r n \mathcal{F}} \delta j^{2}\right. \\
& \left.+\frac{r h \mathcal{H}}{(L-2) \mathcal{F}} \chi^{\prime} \delta j+\frac{\left\{r\left(h^{\prime} \mathcal{H}+2 h \mathcal{H}^{\prime}\right)+4 h \mathcal{H}\right\} f-r f^{\prime} h \mathcal{H}}{2(L-2) f \mathcal{F}} \chi \delta j\right] .
\end{aligned}
$$

Varying Eq. (3.42) with respect to $\delta \mathcal{B}$, the product $\delta j \dot{\delta \mathcal{B}}$ gives the relation $\dot{\delta j}=0$, i.e., Eq. (3.32). Hence the perturbation $\delta j$ is nondynamical. Varying Eq. (3.42) with respect to $\delta j$ and using Eq. (3.25), we obtain the same equation as (3.29). The dynamical perturbation $\chi$ is coupled to $\delta j$ through the last two terms in Eq. (3.42). Indeed, varying Eq. (3.39) with the reduced matter Lagrangian (3.42) in terms of $\chi$ leads to the same equation as (3.33). This shows the consistency of the action approach taken above.

Since the field $\chi$ is coupled to $\delta j$ through the Lagrangians $\chi^{\prime} \delta j$ and $\chi \delta j$, the interaction with $\delta j$ does not affect the propagation of $\chi$ in the limits of large $\omega, k$, and $l$. In this limit, the Lagrangian (3.39) shows that the ghost is absent under the condition $K_{\chi}>0$. This translates to the condition

$$
\mathcal{G}>0 \text {. }
$$

In the approach taken in Sec. III B, we derived the perturbation equations from the original action (3.12), in which case the no-ghost condition (3.43) was not explicit. In the limits of large $\omega, k$, and $l$, the reduced Lagrangian (3.39) leads to the same dispersion relation as Eq. (3.35). Hence the gravitational perturbation $\chi$ has the same radial and angular propagations speeds as those given in Eqs. (3.36) and (3.37), respectively. Combining the no-ghost condition (3.43) with the Laplacian stability conditions $c_{r}^{2} \geq 0$ and $c_{\Omega}^{2} \geq 0$, we require that

$$
\mathcal{F}>0, \quad \mathcal{H}>0
$$

which coincide with those derived in Ref. [71] in the absence of the perfect fluid. The results given above are valid for $l \geq 2$, but the situation is different for $l=1$. Since $L=2$ for $l=1$, the terms proportional to $(L-2)$ vanish from Eq. (3.38). Then, variation of the Lagrangian (3.38) with respect to $W$ gives $\dot{\chi}=0$. Since the property $\dot{\delta j}=0$ also holds, both $\chi$ and $\delta j$ do not propagate for $l=1$.

\section{EVEN-PARITY PERTURBATIONS}

Let us proceed to the derivation of the second-order action and perturbation equations of motion for even-parity modes. The nonvanishing components of metric perturbations in the even-parity sector are given by ${ }^{2}[88]$

$$
h_{t t}=f(r) \sum_{l, m} H_{0}(t, r) Y_{l m}(\theta, \varphi), \quad h_{t r}=h_{r t}=\sum_{l, m} H_{1}(t, r) Y_{l m}(\theta, \varphi), \quad h_{r r}=h(r)^{-1} \sum_{l, m} H_{2}(t, r) Y_{l m}(\theta, \varphi)
$$

\footnotetext{
${ }^{2}$ In Ref. [79], the notation $\alpha$ was used for $h_{1}$.
} 


$$
\begin{aligned}
& h_{t a}=h_{a t}=\sum_{l, m} h_{0}(t, r) \nabla_{a} Y_{l m}(\theta, \varphi), \quad h_{r a}=h_{a r}=\sum_{l, m} h_{1}(t, r) \nabla_{a} Y_{l m}(\theta, \varphi), \\
& h_{a b}=\sum_{l, m}\left[K(t, r) g_{a b} Y_{l m}(\theta, \varphi)+r^{2} G(t, r) \nabla_{a} \nabla_{b} Y_{l m}(\theta, \varphi)\right],
\end{aligned}
$$

where $H_{0}, H_{1}, H_{2}, h_{0}, h_{1}, K$, and $G$ are functions of $t$ and $r$. The scalar field $\phi$ is decomposed into the background value $\bar{\phi}(r)$ and the perturbation $\delta \phi(t, r)$ as

$$
\phi=\bar{\phi}(r)+\sum_{l, m} \delta \phi(t, r) Y_{l m}(\theta, \varphi) .
$$

For the matter sector, the components of $J_{\mu}$ containing even-parity perturbations are expressed in the forms [79]

$$
J_{t}=\bar{J}_{t}+\sum_{l, m} \sqrt{-\bar{g}} \delta J_{t}(t, r) Y_{l m}(\theta, \varphi), \quad J_{r}=\sum_{l, m} \sqrt{-\bar{g}} \delta J_{r}(t, r) Y_{l m}(\theta, \varphi), \quad J_{a}=\sum_{l, m} \sqrt{-\bar{g}} \delta J(t, r) \nabla_{a} Y_{l m}(\theta, \varphi) .
$$

We will mostly omit a bar from the background quantities. The intrinsic spatial vector fields $\mathcal{A}_{i}$ and $\mathcal{B}^{i}$ are given by

$$
\mathcal{A}_{i}=\delta \mathcal{A}_{i}, \quad \mathcal{B}^{i}=x^{i}+\delta \mathcal{B}^{i}
$$

with the perturbed components,

$$
\begin{array}{rlrl}
\delta \mathcal{A}_{r} & =\sum_{l, m} \delta \mathcal{A}_{1}(t, r) Y_{l m}(\theta, \varphi), & \delta \mathcal{A}_{a} & =\sum_{l, m} \delta \mathcal{A}_{2}(t, r) \nabla_{a} Y_{l m}(\theta, \varphi), \\
\delta \mathcal{B}^{r} & =\sum_{l, m} \delta \mathcal{B}_{1}(t, r) Y_{l m}(\theta, \varphi), & \delta \mathcal{B}^{a}=\sum_{l, m} \delta \mathcal{B}_{2}(t, r) \nabla_{a} Y_{l m}(\theta, \varphi) .
\end{array}
$$

Unlike Ref. [79], we define $\delta \mathcal{B}_{1}$ and $\delta \mathcal{B}_{2}$ as the components of $\delta \mathcal{B}^{i}$ (i.e., upper index). The Lagrangian multiplier $\ell$ is decomposed as

$$
\ell=-\rho_{, n}(r) \sqrt{f(r)} t+\sum_{l, m} \delta \ell(t, r) Y_{l m}(\theta, \varphi)
$$

where the first term on the right hand-side is the background value, see Eq. (3.8). The explicit form of $\delta \ell$ is known from Eq. (2.6). Similarly, it is also determined by using the perturbation equation of $J_{\mu}$ derived later. In the following, we will take the latter approach.

The matter density perturbation $\delta \rho(t, r)$ is related to the first-order number density perturbation $\delta n$, as

$$
\delta n=\sum_{l, m} \frac{\delta \rho(t, r)}{\rho_{, n}(r)} Y_{l m}(\theta, \varphi) .
$$

To compute the second-order action of even-parity perturbations, we set $m=0$ without loss of generality. Expanding Eq. (2.5) on account of Eqs. (4.1) and (4.3), the first-order perturbation of $n$ is expressed in the form

$$
\delta n=-\sum_{l}\left[\frac{\delta J_{t}}{\sqrt{f}}+n\left(\frac{1}{2} H_{2}-H_{0}+K-\frac{L}{2} G\right)\right] Y_{l 0}(\theta, \varphi)+\mathcal{O}\left(\varepsilon^{2}\right),
$$

where $\varepsilon^{n}$ represents the $n$-th order of perturbations, and we used the property

$$
Y_{l 0, \theta \theta}+(\cot \theta) Y_{l 0, \theta}+L Y_{l 0}=0
$$

with $L=l(l+1)$. Comparing the first-order term of Eq. (4.9) with Eq. (4.8), it follows that

$$
\delta J_{t}=-\sqrt{f}\left[\frac{\delta \rho}{\rho_{, n}}+n\left(\frac{1}{2} H_{2}-H_{0}+K-\frac{L}{2} G\right)\right] .
$$

We will exploit this relation to eliminate $\delta J_{t}$ from the action. The matter density $\rho(n)$ in Eq. (2.3) is expanded as

$$
\rho(n)=\bar{\rho}(n)+\rho_{, n} \delta n+\frac{c_{m}^{2} \rho_{, n}}{2 n} \delta n^{2}+\mathcal{O}\left(\varepsilon^{3}\right),
$$


where $c_{m}^{2}$ is the matter propagation speed squared defined by

$$
c_{m}^{2} \equiv \frac{n \rho_{, n n}}{\rho_{, n}} .
$$

In Eq. (4.9), we will take the second-order contribution to $\delta n$ into account for the computation of $\rho_{, n} \delta n$.

The other components of $J_{\mu}$, i.e., $\delta J_{r}$ and $\delta J$, can be expressed in terms of the perturbed components of fluid four velocity $u_{\mu}$. We write the radial and angular components of $u_{\mu}$ in the forms

$$
u_{r}=\sum_{l, m} \delta u_{r}(t, r) Y_{l m}(\theta, \varphi), \quad u_{a}=\sum_{l, m} v(t, r) \nabla_{a} Y_{l m}(\theta, \varphi)
$$

where $\delta u_{r}$ and $v$ are functions of $t$ and $r$. Since the relation between $u_{\mu}$ and $J_{\mu}$ is $u_{\mu}=J_{\mu} /(n \sqrt{-g})$, we have

$$
\delta J_{r}=n \delta u_{r}, \quad \delta J=n v .
$$

We will use the perturbations $\delta u_{r}$ and $v$ instead of $\delta J_{r}$ and $\delta J$ in the following discussion.

\section{A. Second-order action}

We expand the action (2.1) up to second order in even-parity perturbations without choosing any particular gauge conditions. The functions $G_{j}(j=2,3,4,5)$ are expressed as

$$
G_{j}(\phi, X)=G_{j}(r)+G_{j, \phi} \delta \phi+G_{j, X} \delta X+\frac{1}{2} G_{j, \phi \phi} \delta \phi^{2}+\frac{1}{2} G_{j, X X} \delta X^{2}+G_{j, \phi X} \delta \phi \delta X+\mathcal{O}\left(\varepsilon^{3}\right),
$$

where $\delta X$ is given by

$$
\begin{aligned}
\delta X= & \sum_{l} \frac{1}{2} h \phi^{\prime}\left(\phi^{\prime} H_{2}-2 \delta \phi^{\prime}\right) Y_{l 0}+\sum_{l} \frac{1}{2 f}\left[\dot{\delta \phi^{2}}+h^{2} \phi^{\prime 2} H_{1}^{2}-h\left\{f \delta \phi^{\prime 2}+2 \phi^{\prime}\left(H_{1} \dot{\delta \phi}-f H_{2} \delta \phi^{\prime}\right)+f \phi^{\prime 2} H_{2}^{2}\right\}\right] Y_{l 0}^{2} \\
& -\sum_{l} \frac{1}{2 r^{2}}\left(\delta \phi-h \phi^{\prime} h_{1}\right)^{2} Y_{l 0, \theta}^{2}+\mathcal{O}\left(\varepsilon^{3}\right) .
\end{aligned}
$$

After expanding the action, we can integrate out the $\theta$-dependent terms by exploiting the integrals (3.10). Then, we perform the integrations by parts with respect to $t$ and $r$ and use the background Eqs. (2.20)-(2.22) and (2.24)-(2.28). After the lengthy but straightforward calculations, the reduced second-order action is expressed in the form

$$
\mathcal{S}_{\text {even }}^{(2)}=\sum_{l} \int \mathrm{d} t \mathrm{~d} r \mathcal{L}_{\text {even }},
$$

where

$$
\begin{aligned}
\mathcal{L}_{\text {even }}= & H_{0}\left[a_{1} \delta \phi^{\prime \prime}+a_{2} \delta \phi^{\prime}+a_{3} H_{2}^{\prime}+L a_{4} h_{1}^{\prime}+\left(a_{5}+L a_{6}\right) \delta \phi+\left(a_{7}+L a_{8}\right) H_{2}+L a_{9} h_{1}+a_{10} \delta \rho\right] \\
& +\left(L b_{1}+\tilde{b}_{1}\right) H_{1}^{2}+H_{1}\left(b_{2} \dot{\delta \phi}+b_{3} \dot{\delta \phi}+b_{4} \dot{H}_{2}+L b_{5} \dot{h}_{1}\right)+c_{1} \dot{\delta \phi} \dot{H}_{2}+H_{2}\left[c_{2} \delta \phi^{\prime}+\left(c_{3}+L c_{4}\right) \delta \phi+L c_{5} h_{1}\right]+c_{6} H_{2}^{2} \\
& +L d_{1} \dot{h}_{1}^{2}+L h_{1}\left(d_{2} \delta \phi^{\prime}+d_{3} \delta \phi\right)+L d_{4} h_{1}^{2}+e_{1} \dot{\delta \phi^{2}}+e_{2} \delta \phi^{\prime 2}+\left(e_{3}+L e_{4}\right) \delta \phi^{2} \\
& +L f_{1} v^{2}+f_{2} \delta \rho^{2}+f_{3} \delta \ell^{\prime} H_{1}+\delta \ell\left(f_{4} \dot{\delta} \rho+f_{5} \dot{H}_{2}+f 6 \delta u_{r}^{\prime}+f_{7} \delta u_{r}+L f_{8} v\right)+f_{9} \delta u_{r}^{2}+f_{10} \delta u_{r} \delta \mathcal{A}_{1}+L f_{11} v \delta \mathcal{A}_{2} \\
& +f_{12} \delta \mathcal{A}_{1} H_{1}+f_{13}\left(\delta \mathcal{A}_{1} \delta \dot{\mathcal{B}}_{1}+L \delta \mathcal{A}_{2} \delta \dot{\mathcal{B}}_{2}\right) \\
& +g_{1}\left(L \dot{G}^{\prime}-2 \dot{K}^{\prime}\right) H_{1}+g_{2}\left(L G^{\prime \prime}-2 K^{\prime \prime}\right) H_{0}+g_{3}\left(L \dot{G}^{2}+2 \dot{K}^{2}\right)+g_{4}\left(L G^{\prime 2}+2 K^{\prime 2}\right)+L g_{5} \dot{G} \dot{K}+L g_{6} G^{\prime} K^{\prime} \\
& +(L \dot{G}-2 \dot{K})\left(g_{7} \dot{H}_{2}+g_{8} \dot{\delta \phi}+g_{9} H_{1}+g_{10} \delta \ell\right)+L g_{11}(\dot{G}-\dot{K}) h_{0} \\
& +\left(L G^{\prime}-2 K^{\prime}\right)\left(g_{12} \delta \phi^{\prime}+g_{13} H_{0}+g_{14} H_{2}+g_{15} \delta \phi\right)+L g_{16}\left(G^{\prime}-K^{\prime}\right) h_{1}+(L-2) K\left(k_{1} H_{0}+k_{2} H_{2}+k_{3} \delta \phi\right) \\
& +L\left[m_{1} h_{0}^{\prime 2}+m_{2} h_{0}^{\prime}\left(\dot{h}_{1}+H_{1}\right)+m_{3} h_{0}^{2}+h_{0}\left\{m_{4} \dot{H}_{2}+m_{5} \dot{h}_{1}+m_{6} \dot{\delta \phi}+m_{7} H_{1}+m_{8}\left(\delta \ell+\delta \mathcal{A}_{2}\right)\right\}\right] .
\end{aligned}
$$

The coefficients $a_{1}, a_{2}, \ldots$ etc, which depend on the background quantities, are explicitly given in Appendix $\mathrm{A}^{3}$.

\footnotetext{
${ }^{3}$ By using the Maple software, we confirmed that the calculations independently performed by the authors agree with each other.
} 


\section{B. Perturbation equations of motion}

From the action (4.18), we will derive the full linear perturbation equations of motion without fixing any particular gauge conditions. For the coefficients $a_{10}, \tilde{b}_{1}, f_{i}(i=1, \ldots, 13), g_{10}$, and $m_{8}$, we use their explicit forms given in Appendix A.

We first derive the perturbation equations associated with the perfect fluid. Varying the action (4.18) with respect to $v$, we obtain

$$
\delta \ell=\frac{\rho+P}{n} v-\delta \mathcal{A}_{2} .
$$

This relation will be used to eliminate $\delta \ell$ and its derivatives from the other perturbation equations. Variation of the action (4.18) with respect to $\delta \ell, \delta \rho, \delta u_{r}, \delta \mathcal{A}_{1}, \delta \mathcal{A}_{2}, \delta \mathcal{B}_{1}$, and $\delta \mathcal{B}_{2}$ lead, respectively, to

$$
\begin{aligned}
& \frac{\dot{\delta} \rho}{\rho+P}+\frac{1}{2} \dot{H}_{2}+\dot{K}-\frac{1}{2} L \dot{G}-h H_{1}^{\prime}+h \sqrt{f} \delta u_{r}^{\prime}+\left(\frac{1}{2} \frac{f^{\prime} h}{f c_{m}^{2}}-\frac{h^{\prime}}{2}-\frac{2 h}{r}\right) H_{1}+\frac{L}{r^{2}} h_{0} \\
& +\left[\frac{1}{2} \frac{h\left(c_{m}^{2}-1\right) f^{\prime}}{\sqrt{f} c_{m}^{2}}+\frac{\sqrt{f} h^{\prime}}{2}+\frac{2 h \sqrt{f}}{r}\right] \delta u_{r}-\frac{L \sqrt{f}}{r^{2}} v=0, \\
& \frac{c_{m}^{2}}{\rho+P} \delta \rho-\frac{1}{2} H_{0}+\frac{\dot{v}}{\sqrt{f}}-\frac{n \delta \dot{\mathcal{A}}_{2}}{\sqrt{f}(\rho+P)}=0, \\
& \delta u_{r}-v^{\prime}+\frac{f^{\prime}}{2 f} v-\frac{n}{\rho+P}\left(\delta \mathcal{A}_{1}-\delta \mathcal{A}_{2}^{\prime}\right)=0, \\
& \delta \dot{\mathcal{B}}_{1}+h\left(\sqrt{f} \delta u_{r}-H_{1}\right)=0 \\
& \delta \dot{\mathcal{B}}_{2}-\frac{h_{0}-v \sqrt{f}}{r^{2}}=0 \\
& \delta \dot{\mathcal{A}}_{1}=0 \\
& \delta \dot{\mathcal{A}}_{2}=0 .
\end{aligned}
$$

From Eqs. (4.26) and (4.27), the Lagrange multipliers $\delta \mathcal{A}_{1}$ and $\delta \mathcal{A}_{2}$ depend on the distance $r$ alone. The last term on the left hand-side of Eq. (4.22) vanishes due to the property $\delta \dot{\mathcal{A}}_{2}=0$. In the following, we also employ the relations (4.26) and (4.27) for other perturbation equations of motion. Taking the time derivative of Eq. (4.23) and differentiating the resulting equation with respect to $r$ further, we have

$$
\begin{aligned}
& \dot{\delta u_{r}}-\dot{v}^{\prime}+\frac{f^{\prime}}{2 f} \dot{v}=0, \\
& \dot{\delta} u_{r}^{\prime}-\dot{v}^{\prime \prime}+\frac{f^{\prime}}{2 f} \dot{v}^{\prime}+\frac{1}{2}\left(\frac{f^{\prime \prime}}{f}-\frac{f^{\prime 2}}{f^{2}}\right) \dot{v}=0 .
\end{aligned}
$$

The Lagrange multipliers $\delta \mathcal{B}_{1}$ and $\delta \mathcal{B}_{2}$ are related to the components of fluid four velocity through Eqs. (4.24) and (4.25).

Varying the action (4.18) with respect to the metric perturbations $H_{0}, H_{1}, H_{2}, h_{0}, h_{1}$ and $K$, the resulting perturbation equations are given, respectively, by

$$
\begin{aligned}
& a_{1} \delta \phi^{\prime \prime}+a_{2} \delta \phi^{\prime}+a_{3} H_{2}^{\prime}+L a_{4} h_{1}^{\prime}+\left(a_{5}+L a_{6}\right) \delta \phi+\left(a_{7}+L a_{8}\right) H_{2}+L a_{9} h_{1}+\frac{1}{2} \frac{r^{2} \sqrt{f}}{\sqrt{h}} \delta \rho \\
& +g_{2}\left(L G^{\prime \prime}-2 K^{\prime \prime}\right)+g_{13}\left(L G^{\prime}-2 K^{\prime}\right)+(L-2) k_{1} K=0, \\
& {\left[2 L b_{1}-\frac{r^{2}(\rho+P) \sqrt{h}}{\sqrt{f}}\right] H_{1}+b_{2} \dot{\delta \phi}^{\prime}+b_{3} \dot{\delta \phi}+b_{4} \dot{H}_{2}+L b_{5} \dot{h}_{1}+r^{2}(\rho+P) \sqrt{h} \delta u_{r}} \\
& +g_{1}\left(L \dot{G}^{\prime}-2 \dot{K}^{\prime}\right)+g_{9}(L \dot{G}-2 \dot{K})+L m_{2} h_{0}^{\prime}+L m_{7} h_{0}=0, \\
& -c_{1} \ddot{\delta \phi}-b_{4} \dot{H}_{1}+c_{2} \delta \phi^{\prime}+\left(c_{3}+L c_{4}\right) \delta \phi+L c_{5} h_{1}+2 c_{6} H_{2}-a_{3} H_{0}^{\prime}+\left(L a_{8}+a_{7}-a_{3}^{\prime}\right) H_{0}-\frac{1}{2} \frac{r^{2}(\rho+P)}{\sqrt{h}} \dot{v} \\
& -g_{7}(L \ddot{G}-2 \ddot{K})+g_{14}\left(L G^{\prime}-2 K^{\prime}\right)+k_{2}(L-2) K-L m_{4} \dot{h}_{0}=0, \\
& -2 L m_{1} h_{0}^{\prime \prime}-2 L m_{1}^{\prime} h_{0}^{\prime}-L m_{2}\left(\dot{h}_{1}^{\prime}+H_{1}^{\prime}\right)+2 L m_{3} h_{0}+L m_{4} \dot{H}_{2}+\left(m_{5}-m_{2}^{\prime}\right) L \dot{h}_{1}+L m_{6} \dot{\delta} \phi+\left(m_{7}-m_{2}^{\prime}\right) L H_{1}
\end{aligned}
$$




$$
\begin{aligned}
& +\frac{(\rho+P) L}{\sqrt{h}} v+L g_{11}(\dot{G}-\dot{K})=0 \\
& -2 d_{1} \ddot{h}_{1}+d_{2} \delta \phi^{\prime}+d_{3} \delta \phi+2 d_{4} h_{1}-a_{4} H_{0}^{\prime}+\left(a_{9}-a_{4}^{\prime}\right) H_{0}-b_{5} \dot{H}_{1}+c_{5} H_{2}-m_{2} \dot{h}_{0}^{\prime}-m_{5} \dot{h}_{0}+g_{16}\left(G^{\prime}-K^{\prime}\right)=0,(4 \\
& -2 g_{1} \dot{H}_{1}^{\prime}-2 g_{2} H_{0}^{\prime \prime}-4 g_{3} \ddot{K}-4 g_{4} K^{\prime \prime}-4 g_{4}^{\prime} K^{\prime}-L g_{5} \ddot{G}-L g_{6} G^{\prime \prime}-L g_{6}^{\prime} G^{\prime}+2 g_{7} \ddot{H}_{2}+2 g_{8} \ddot{\delta} \phi+2\left(g_{9}-g_{1}^{\prime}\right) \dot{H}_{1} \\
& +L g_{11} \dot{h}_{0}+2 g_{12} \delta \phi^{\prime \prime}+2\left(g_{13}-2 g_{2}^{\prime}\right) H_{0}^{\prime}+2 g_{14} H_{2}^{\prime}+2\left(g_{15}+g_{12}^{\prime}\right) \delta \phi^{\prime}+L g_{16} h_{1}^{\prime}+L g_{16}^{\prime} h_{1} \\
& +\left[(L-2) k_{3}+2 g_{15}^{\prime}\right] \delta \phi+\left[(L-2) k_{1}-2 g_{2}^{\prime \prime}+2 g_{13}^{\prime}\right] H_{0}+\left[(L-2) k_{2}+2 g_{14}^{\prime}\right] H_{2}-\frac{r^{2}(\rho+P)}{\sqrt{h}} \dot{v}=0
\end{aligned}
$$

For the derivation of Eq. (4.31), we used Eq. (4.23) to eliminate the combination $\delta \mathcal{A}_{1}-\delta \mathcal{A}_{2}^{\prime}$ of Lagrange multipliers.

Varying the action (4.18) with respect to $G$ and combining the resulting equation with Eq. (4.35), it follows that

$$
\begin{aligned}
& -2\left(2 g_{3}+g_{5}\right) \ddot{K}-\left(4 g_{3}+L g_{5}\right) \ddot{G}-2\left(2 g_{4}+g_{6}\right) K^{\prime \prime}-\left(4 g_{4}+L g_{6}\right) G^{\prime \prime}-2\left(2 g_{4}^{\prime}+g_{6}^{\prime}\right) K^{\prime}-\left(4 g_{4}^{\prime}+L g_{6}^{\prime}\right) G^{\prime} \\
& +(L-2)\left(g_{11} \dot{h}_{0}+g_{16} h_{1}^{\prime}+g_{16}^{\prime} h_{1}+k_{1} H_{0}+k_{2} H_{2}+k_{3} \delta \phi\right)=0 .
\end{aligned}
$$

Finally, variation of the action (4.18) with respect to $\delta \phi$ leads to

$$
\begin{aligned}
& -2 e_{1} \ddot{\delta} \phi-2 e_{2} \delta \phi^{\prime \prime}+2\left(e_{3}+L e_{4}\right) \delta \phi+a_{1} H_{0}^{\prime \prime}+\left(2 a_{1}^{\prime}-a_{2}\right) H_{0}^{\prime}+\left(a_{1}^{\prime \prime}-a_{2}^{\prime}+a_{5}+L a_{6}\right) H_{0}+b_{2} \dot{H}_{1}^{\prime}+\left(b_{2}^{\prime}-b_{3}\right) \dot{H}_{1} \\
& -c_{1} \ddot{H}_{2}-c_{2} H_{2}^{\prime}-\left(c_{2}^{\prime}-c_{3}-L c_{4}\right) H_{2}-L d_{2} h_{1}^{\prime}+L\left(d_{3}-d_{2}^{\prime}\right) h_{1}-2 e_{2}^{\prime} \delta \phi^{\prime}+g_{8}(2 \ddot{K}-L \ddot{G})+g_{12}\left(2 K^{\prime \prime}-L G^{\prime \prime}\right) \\
& -\left(g_{15}-g_{12}^{\prime}\right)\left(2 K^{\prime}-L G^{\prime}\right)+k_{3}(L-2) K-L m_{6} \dot{h}_{0}=0 .
\end{aligned}
$$

We thus derived the full set of linear perturbation equations of motion in the gauge-ready form. We note that Eqs. (4.30)-(4.37) do not contain the Lagrange multipliers $\delta \mathcal{A}_{i}$ and $\delta \mathcal{B}_{i}$.

\section{PROPAGATION OF EVEN-PARITY PERTURBATIONS}

To study the propagation of even-parity perturbations, we fix the residual gauge degrees of freedom. We consider the infinitesimal gauge transformation $x_{\mu} \rightarrow x_{\mu}+\xi_{\mu}$, where

$$
\xi_{t}=\sum_{l, m} \mathcal{T}(t, r) Y_{l m}(\theta, \varphi), \quad \xi_{r}=\sum_{l, m} \mathcal{R}(t, r) Y_{l m}(\theta, \varphi), \quad \xi_{a}=\sum_{l, m} \Theta(t, r) \nabla_{a} Y_{l m}(\theta, \varphi) .
$$

Then, the transformations of even-parity perturbations $H_{0}, H_{1}, H_{2}, h_{0}, h_{1}, K, G$, and $\delta \phi$ are given, respectively, by $[72,91]$

$$
\begin{aligned}
& H_{0} \rightarrow H_{0}+\frac{2}{f} \dot{\mathcal{T}}-\frac{f^{\prime} h}{f} \mathcal{R}, \quad H_{1} \rightarrow H_{1}+\dot{\mathcal{R}}+\mathcal{T}^{\prime}-\frac{f^{\prime}}{f} \mathcal{T}, \quad H_{2} \rightarrow H_{2}+2 h \mathcal{R}^{\prime}+h^{\prime} \mathcal{R}, \\
& h_{0} \rightarrow h_{0}+\mathcal{T}+\dot{\Theta}, \quad h_{1} \rightarrow h_{1}+\mathcal{R}+\Theta^{\prime}-\frac{2}{r} \Theta, \quad K \rightarrow K+\frac{2}{r} h \mathcal{R}, \quad G \rightarrow G+\frac{2}{r^{2}} \Theta, \\
& \delta \phi \rightarrow \delta \phi-\phi^{\prime} h \mathcal{R} .
\end{aligned}
$$

It is possible to construct gauge-invariant quantities invariant under the above gauge transformation. One can combine $G$ and its derivatives with other perturbed quantities to eliminate $\Theta$. In the same manner, the transformation scalar $\mathcal{T}$ can be removed by combining perturbed quantities with $h_{0}-r^{2} \dot{G} / 2$ and its derivatives. For the remaining transformation scalar $\mathcal{R}$, there are several ways to eliminate it. If we use $K$, for instance, we can construct the several gauge-invariant variables:

$$
\begin{aligned}
& \tilde{H}_{0}=H_{0}-\frac{2}{f} \frac{\partial}{\partial t}\left(h_{0}-\frac{r^{2}}{2} \dot{G}\right)+\frac{r f^{\prime}}{2 f} K, \quad \tilde{H}_{1}=H_{1}-f\left[\frac{1}{f}\left(h_{0}-\frac{r^{2}}{2} \dot{G}\right)\right]^{\prime}-\frac{r}{2 h} \dot{K}, \\
& \tilde{H}_{2}=H_{2}-\sqrt{h}\left(\frac{r K}{\sqrt{h}}\right)^{\prime}, \quad \tilde{h}_{1}=h_{1}-\frac{r^{2}}{2} G^{\prime}-\frac{r}{2 h} K, \quad \tilde{\delta \phi}=\delta \phi+\frac{r \phi^{\prime}}{2} K .
\end{aligned}
$$

In the later discussion, we will consider the following gauge-invariant combination

$$
\psi=\tilde{H}_{2}+\frac{a_{4}}{a_{3}} L \tilde{h}_{1}+\frac{a_{1}}{a_{3}} \tilde{\delta \phi^{\prime}} .
$$


For $l \geq 2$, we can fix the transformation scalars $\mathcal{T}$ and $\Theta$ by choosing the gauge:

$$
h_{0}=0, \quad G=0 .
$$

In order to fix the other transformation scalar $\mathcal{R}$, Regge and Wheeler [88] chose the spatially diagonal gauge $h_{1}=0$. The other possible choices are the uniform curvature gauge $K=0$ and the unitary gauge $\delta \phi=0$. Following the references $[72,90,91]$ of even-parity perturbations in modified gravity theories, we choose the gauge

$$
K=0 .
$$

Then, the gauge-invariant variables in Eq. (5.3) reduce to $\tilde{H}_{2}=H_{2}, \tilde{h}_{1}=h_{1}$, and $\tilde{\delta \phi}=\delta \phi$. For this gauge choice, the quantity (5.4) yields

$$
\psi=H_{2}+\frac{a_{4}}{a_{3}} L h_{1}+\frac{a_{1}}{a_{3}} \delta \phi^{\prime},
$$

which corresponds to that introduced in Ref. [72].

In Horndeski theories with matter, there are three propagating degrees of freedom in the even-parity sector. The first is the fluid density perturbation $\delta \rho$, and the second is the scalar-field perturbation $\delta \phi$. The third is the perturbation $\psi$, which corresponds to the propagating degree of freedom arising from the gravity sector. From Eq. (5.7), we replace $\mathrm{H}_{2}$ with $\psi$ to study the propagation of the dynamical degrees of freedom. We derive second-order differential equations of the three dynamical variables $\delta \rho, \delta \phi, \psi$ and study the propagation of them along the radial and angular directions. For this purpose, we need to eliminate the other non-dynamical variables from the perturbation equations presented in Sec. IV B. In the following, we first investigate the case $l \geq 2$ and then finally study the $l=0$ and $l=1$ cases separately.

\section{A. $l \geq 2$}

To reduce the number of nondynamical degrees of freedom from the perturbation equations of motion for $l \geq 2$, we first take the time derivative of Eq. (4.21) and eliminate $\dot{\delta u_{r}}$ and $\dot{\delta u_{r}^{\prime}}$ on account of Eqs. (4.28) and (4.29). This process gives rise to the third derivative $\dot{v}^{\prime \prime}$ as well as $\dot{v}^{\prime}, \dot{v}$. From Eqs. (4.22) and (4.27), we have

$$
\dot{v}=-\sqrt{f}\left(\frac{c_{m}^{2}}{\rho+P} \delta \rho-\frac{1}{2} H_{0}\right) .
$$

Taking the $r$ derivative of this relation twice, the third derivative $\dot{v}^{\prime \prime}$ is replaced with the second derivatives $\delta \rho^{\prime \prime}$ and $H_{0}^{\prime \prime}$. Indeed, this procedure generates a Laplacian term proportional to $c_{m}^{2} \delta \rho^{\prime \prime}$. As a consequence, we obtain the perturbation equation containing up to the second derivatives of $\delta \rho$, as

$$
\begin{aligned}
& \frac{2 \ddot{\delta} \rho}{\rho+P}+\ddot{\psi}-\frac{2 f h c_{m}^{2}}{\rho+P} \delta \rho^{\prime \prime}-\left[8 f h c_{m} c_{m}^{\prime}+\left(4 c_{m}^{2}+1\right) h f^{\prime}+c_{m}^{2} f h^{\prime}+\frac{4 f h c_{m}^{2}}{r}\right] \frac{\delta \rho^{\prime}}{\rho+P} \\
& +\left[\frac{2 f c_{m}^{2} L}{r^{2}}-4 f h\left(c_{m} c_{m}^{\prime \prime}+c_{m}^{\prime 2}\right)-2 f h\left(\frac{4 f^{\prime}}{f}+\frac{h^{\prime}}{h}+\frac{4}{r}\right) c_{m} c_{m}^{\prime}-\frac{1}{2} f^{\prime} h\left(\frac{f^{\prime}}{f}+\frac{h^{\prime}}{h}+\frac{4}{r}+\frac{2 f^{\prime \prime}}{f^{\prime}}\right)\left(1+c_{m}^{2}\right)\right] \frac{\delta \rho}{\rho+P} \\
& -\frac{L a_{4} \ddot{h}_{1}}{a_{3}}+\left(\frac{f^{\prime} h}{f c_{m}^{2}}-h^{\prime}-\frac{4 h}{r}\right) \dot{H}_{1}+\left[\frac{h\left(2 c_{m}^{2}-1\right) f^{\prime}}{2 c_{m}^{2}}+\frac{f h^{\prime}}{2}+\frac{2 f h}{r}\right] H_{0}^{\prime}-\frac{f}{r^{2}} L H_{0}-\frac{a_{1} \ddot{\delta \phi}^{\prime}-h a_{3}\left(f H_{0}^{\prime \prime}-2 \dot{H}_{1}^{\prime}\right)}{a_{3}}=0,(5.9
\end{aligned}
$$

where we used the property $\rho^{\prime}=(\rho+P) n^{\prime} / n$ with $n^{\prime}=-n f^{\prime} /\left(2 f c_{m}^{2}\right)$. This equation still contains the third derivative $\ddot{\delta} \phi^{\prime}$, but it can be removed by using other perturbation equations of motion. Indeed, the same higher-order term also appears in Eqs. (4.35) and (4.37) from the term $\ddot{H}_{2}$ by taking the second time derivative of Eq. (5.7). These equations 
are given, respectively, by

$$
\begin{aligned}
& g_{7} \ddot{\psi}+g_{8} \ddot{\delta \phi}-\frac{a_{1} g_{14}-a_{3} g_{12}}{a_{3}} \delta \phi^{\prime \prime}+g_{14} \psi^{\prime}-\left[\frac{1}{2} \frac{a_{1} k_{2} L}{a_{3}}-g_{12}^{\prime}-g_{15}-\frac{\left(k_{2}-g_{14}^{\prime}\right) a_{1}-g_{14} a_{1}^{\prime}}{a_{3}}-\frac{a_{1} a_{3}^{\prime} g_{14}}{a_{3}{ }^{2}}\right] \delta \phi^{\prime} \\
& +\left(\frac{1}{2} k_{2} L-k_{2}+g_{14}^{\prime}\right) \psi+\left(\frac{1}{2} k_{3} L-k_{3}+g_{15}^{\prime}\right) \delta \phi+\frac{c_{m}^{2} r^{2} \sqrt{f}}{2 \sqrt{h}} \delta \rho-\frac{g_{7} a_{4}}{a_{3}} L \ddot{h}_{1}+\left(g_{9}-g_{1}^{\prime}\right) \dot{H}_{1}+\left(g_{13}-2 g_{2}^{\prime}\right) H_{0}^{\prime} \\
& -\left(\frac{g_{14} a_{4}}{a_{3}}-\frac{g_{16}}{2}\right) L h_{1}^{\prime}+\left[\frac{1}{2} k_{1} L-k_{1}+g_{13}^{\prime}-g_{2}^{\prime \prime}-\frac{(\rho+P) r^{2} \sqrt{f}}{4 \sqrt{h}}\right] H_{0} \\
& -\left[\frac{1}{2} \frac{a_{4} k_{2} L}{a_{3}}-\frac{g_{16}^{\prime}}{2}+\frac{\left(g_{14}^{\prime}-k_{2}\right) a_{4}+g_{14} a_{4}^{\prime}}{a_{3}}-\frac{g_{14} a_{3}^{\prime} a_{4}}{a_{3}^{2}}\right] L h_{1}-\frac{1}{2} \frac{r^{2} a_{4}\left[a_{1} \ddot{\delta \phi}-h a_{3}\left(f H_{0}^{\prime \prime}-2 \dot{H}_{1}^{\prime}\right)\right]}{f h a_{3}}=0 \\
& e_{1} \ddot{\delta \phi}+\frac{c_{1}}{2} \ddot{\psi}+\left(e_{2}-\frac{1}{2} \frac{c_{2} a_{1}}{a_{3}}\right) \delta \phi^{\prime \prime}+\left[\frac{1}{2} \frac{c_{4} a_{1} L}{a_{3}}+e_{2}^{\prime}+\frac{1}{2} \frac{\left(c_{3}-c_{2}^{\prime}\right) a_{1}-c_{2} a_{1}^{\prime}}{a_{3}}+\frac{1}{2} \frac{c_{2} a_{1} a_{3}^{\prime}}{a_{3}{ }^{2}}\right] \delta \phi^{\prime} \\
& +\frac{c_{2}}{2} \psi^{\prime}-\left(e_{3}+L e_{4}\right) \delta \phi-\frac{1}{2}\left(L c_{4}+c_{3}-c_{2}^{\prime}\right) \psi-\frac{c_{1} a_{4}}{2 a_{3}} L \ddot{h_{1}}+\frac{1}{2}\left(b_{3}-b_{2}^{\prime}\right) \dot{H}_{1}+\left(\frac{a_{2}}{2}-a_{1}^{\prime}\right) H_{0}^{\prime} \\
& +\frac{1}{2}\left(d_{2}-\frac{c_{2} a_{4}}{a_{3}}\right) L h_{1}^{\prime}-\frac{1}{2}\left(L a_{6}+a_{5}-a_{2}^{\prime}+a_{1}^{\prime \prime}\right) H_{0} \\
& +\frac{1}{2}\left[\frac{c_{4} a_{4} L}{a_{3}}-d_{3}+d_{2}^{\prime}+\frac{\left(c_{3}-c_{2}^{\prime}\right) a_{4}-c_{2} a_{4}^{\prime}}{a_{3}}+\frac{c_{2} a_{3}^{\prime} a_{4}}{a_{3}^{2}}\right] L h_{1}+\frac{1}{2} \frac{a_{1}\left[a_{1} \ddot{\delta \phi} \phi^{\prime}-h a_{3}\left(f H_{0}^{\prime \prime}-2 \dot{H}_{1}^{\prime}\right)\right]}{f h a_{3}}=0
\end{aligned}
$$

where we eliminated the term $\dot{v}$ in Eq. (4.35) by using Eq. (5.8). Under the gauge choice (5.5)-(5.6), Eqs. (4.30), (4.32), (4.34), (4.36) reduce, respectively, to

$$
\begin{aligned}
& {\left[\frac{a_{4} a_{8} L}{a_{3}}-a_{9}+a_{4}^{\prime}+\frac{a_{4}\left(a_{7}-a_{3}^{\prime}\right)}{a_{3}}\right] L h_{1}} \\
& =\psi^{\prime} a_{3}-\left[\frac{a_{1} a_{8} L}{a_{3}}-a_{2}+a_{1}^{\prime}+\frac{a_{1}\left(a_{7}-a_{3}^{\prime}\right)}{a_{3}}\right] \delta \phi^{\prime}+\left(L a_{6}+a_{5}\right) \delta \phi+\left(L a_{8}+a_{7}\right) \psi+\frac{1}{2} \frac{r^{2} \sqrt{f} \delta \rho}{\sqrt{h}}, \\
& \frac{a_{1} \ddot{\delta \phi}-h a_{3}\left(f H_{0}^{\prime}-2 \dot{H}_{1}\right)}{f h}+\left[L a_{8}-a_{3}^{\prime}+a_{7}-\frac{(\rho+P) r^{2} \sqrt{f}}{4 \sqrt{h}}\right] H_{0}+\left(c_{5}-\frac{2 c_{6} a_{4}}{a_{3}}\right) L h_{1} \\
& =\left(\frac{2 c_{6} a_{1}}{a_{3}}-c_{2}\right) \delta \phi^{\prime}-\left(L c_{4}+c_{3}\right) \delta \phi-2 c_{6} \psi-\frac{c_{m}^{2} r^{2} \sqrt{f} \delta \rho}{2 \sqrt{h}}, \\
& \frac{a_{4}}{f}\left(\ddot{h}_{1}+f H_{0}^{\prime}-\dot{H}_{1}\right)+\left(a_{4}^{\prime}-a_{9}\right) H_{0}+\left(\frac{a_{4} c_{5} L}{a_{3}}-2 d_{4}\right) h_{1}=\left(d_{2}-\frac{a_{1} c_{5}}{a_{3}}\right) \delta \phi^{\prime}+d_{3} \delta \phi+\psi c_{5}, \\
& k_{1} H_{0}+g_{16} h_{1}^{\prime}-\left(\frac{a_{4} k_{2} L}{a_{3}}-g_{16}^{\prime}\right) h_{1}=\frac{a_{1} k_{2}}{a_{3}} \delta \phi^{\prime}-k_{3} \delta \phi-k_{2} \psi .
\end{aligned}
$$

Now, we will derive the second-order coupled differential equations for $\delta \rho, \psi$, and $\delta \phi$ by eliminating nondynamical variables $H_{0}, H_{1}, H_{2}, h_{1}$ and their derivatives present in Eqs. (5.9)-(5.15). The second time derivative of $h_{1}$ in Eqs. (5.9)-(5.11) can be eliminated by solving Eq. (5.14) for $\ddot{h}_{1}$. Taking the $r$ derivative of Eq. (5.13), we can remove the combination $a_{1} \ddot{\delta \phi} \phi^{\prime}-h a_{3}\left(f H_{0}^{\prime \prime}-2 \dot{H}_{1}^{\prime}\right)$ appearing in Eqs. (5.9)-(5.11). After this procedure, we eliminate the term $f H_{0}^{\prime}-2 \dot{H}_{1}$ by using Eq. (5.13). The next step is to solve Eq. (5.12) for $h_{1}$ and to take its $r$ derivative. After substituting $h_{1}$ and $h_{1}^{\prime}$ into Eq. (5.15), we solve this equation for $H_{0}$. On using these relations, we can remove the nondynamical variables $h_{1}^{\prime}, H_{0}$, and $h_{1}$ from Eqs. (5.9)-(5.11). Then, we finally end up with the second-order differential equations containing only the dynamical perturbations $\delta \rho, \psi, \delta \phi$ and their derivatives. This is expressed in the form

$$
\boldsymbol{K} \ddot{\overrightarrow{\mathcal{X}}}+G \overrightarrow{\mathcal{X}}^{\prime \prime}+\boldsymbol{Q} \overrightarrow{\mathcal{X}}^{\prime}+\boldsymbol{M} \overrightarrow{\mathcal{X}}=0
$$

where $\boldsymbol{K}, \boldsymbol{G}, \boldsymbol{Q}, \boldsymbol{M}$ are $3 \times 3$ matrices, with

$$
\overrightarrow{\mathcal{X}}=\left(\begin{array}{c}
\delta \rho \\
\psi \\
\delta \phi
\end{array}\right)
$$

In the following, we will investigate the propagation of dynamical perturbations in both radial and angular directions. 


\section{Radial propagation}

The dispersion relation along the radial direction can be derived by assuming the solutions of Eq. (5.17) to be $\overrightarrow{\mathcal{X}}=\overrightarrow{\mathcal{X}}_{0} e^{i(\omega t-k r)}$, where $\overrightarrow{\mathcal{X}}_{0}$ is a constant vector, and $\omega$ and $k$ are the constant frequency and wavenumber respectively. In the limits $\omega \rightarrow \infty$ and $k \rightarrow \infty$, the dominant contributions to Eq. (5.16) are the first two terms. To have nonvanishing solutions of $\overrightarrow{\mathcal{X}}$, we require that $\operatorname{det}\left|\omega^{2} \boldsymbol{K}+k^{2} \boldsymbol{G}\right|=0$. The radial propagation speed in the coordinates $r$ and $t$ is given by $\hat{c}_{r}=\mathrm{d} r / \mathrm{d} t=\omega / k$. We consider the propagation speed $c_{r}$ measured by the rescaled radial coordinate $r_{*}=\int \mathrm{d} r / \sqrt{h}$ and proper time $\tau=\int \sqrt{f} \mathrm{~d} t$. In this case we have $c_{r}=\mathrm{d} r_{*} / \mathrm{d} \tau=\hat{c}_{r} / \sqrt{f h}$, so the dispersion relation translates to

$$
\operatorname{det}\left|f h c_{r}^{2} \boldsymbol{K}+\boldsymbol{G}\right|=0 .
$$

The matrix components of $\boldsymbol{K}$ and $\boldsymbol{G}$ have the following properties

$$
\begin{array}{ll}
K_{21}=0, & K_{31}=0, \\
G_{21}=0, & G_{31}=0 .
\end{array}
$$

Then, the matter propagation speed $c_{r 1}$ along the radial direction decouples from the other two, so that

$$
f h c_{r 1}^{2} K_{11}+G_{11}=0,
$$

where

$$
K_{11}=\frac{2}{\rho+P}, \quad G_{11}=-\frac{2 f h}{\rho+P} c_{m}^{2}
$$

Hence we obtain

$$
c_{r 1}^{2}=c_{m}^{2}
$$

The matter perturbation $\delta \rho$ propagates with the sound speed $c_{m}$. This is different from the value $c_{r 1}^{2}=0$ obtained in Ref. [79], but the latter arises from inappropriate treatment for deriving a reduced matter Lagrangian. The correct radial propagation speed squared along the radial direction is given by Eq. (5.23). In Appendix B, we will see that this difference stems from how to integrate out nondynamical perturbations from the action (analogous to the discussion of odd-parity perturbations in Sec. III B).

From Eq. (5.18), the other two propagation speeds are obtained by solving

$$
\left(K_{22} K_{33}-K_{23} K_{32}\right) f^{2} h^{2} c_{r}^{4}+\left(K_{22} G_{33}+K_{33} G_{22}-K_{23} G_{32}-K_{32} G_{23}\right) f h c_{r}^{2}+G_{22} G_{33}-G_{23} G_{32}=0 .
$$

Although the nonvanishing components of matrices $\boldsymbol{K}$ and $\boldsymbol{G}$ are complicated, the combination of terms appearing in Eq. (5.24) can be simplified in the following way. On using the relations among the quantities given in Appendix A, all of them can be expressed in terms of $a_{1}, a_{4}, c_{2}, c_{3}, c_{4}, e_{3}, \mathcal{F}, \mathcal{G}$, and the derivatives of them. In addition to these variables, we introduce the following combination $[72,79]$,

$$
\mathcal{P}_{1} \equiv \frac{h \mu}{2 f r^{2} \mathcal{H}^{2}}\left(\frac{f r^{4} \mathcal{H}^{4}}{\mu^{2} h}\right)^{\prime}
$$

with

$$
\mu=-\frac{4 a_{3}}{\sqrt{f h}}=\frac{2\left(\phi^{\prime} a_{1}+2 r a_{4}\right)}{\sqrt{f h}}, \quad \mathcal{H}=\frac{2 a_{4}}{\sqrt{f h}},
$$

where $\mathcal{H}$ is the same quantity as defined in Eq. (3.14). We will replace the derivative $a_{1}^{\prime}$ with $\mathcal{P}_{1}$. From the background Eqs. (2.20) and (2.22), there is the following relation

$$
a_{4}^{\prime}=\frac{1}{2 f-r f^{\prime}}\left[\left(r f^{\prime \prime}-\frac{r f^{\prime 2}}{f}+2 f^{\prime}-\frac{2 f}{r}\right) a_{4}+\frac{r f^{3 / 2}}{\sqrt{h}}\left(\frac{\mathcal{F}}{r^{2}}-\rho-P\right)\right],
$$

which will be used to eliminate the term $a_{4}^{\prime}$. As a result, Eq. (5.24) is factorized in the form,

$$
\left(c_{r}^{2}-c_{r 2}^{2}\right)\left(c_{r}^{2}-c_{r 3}^{2}\right)=0,
$$


whose solutions to $c_{r}^{2}$ are given by

$$
\begin{aligned}
c_{r 2}^{2} & =\frac{\mathcal{G}}{\mathcal{F}}, \\
c_{r 3}^{2} & =\frac{4 \phi^{\prime}\left[8 r^{2} f h a_{4} c_{4}\left(\phi^{\prime} a_{1}+r a_{4}\right)-a_{1}^{2} f^{3 / 2} \phi^{\prime} \mathcal{G} \sqrt{h}+2\left(a_{1} f^{\prime}+2 c_{2} f\right) a_{4}^{2} r^{2}\right]}{f^{5 / 2} \sqrt{h}\left[\left(2 \mathcal{P}_{1}-\mathcal{F}\right) h \mu^{2}-2 \mathcal{H}^{2} r^{4}(\rho+P)\right]} .
\end{aligned}
$$

The value $c_{r 2}$, which is equivalent to the radial propagation speed (3.36) in the odd-parity sector, corresponds to the speed of gravitational perturbation $\psi$. We also note that $c_{r 2}$ coincides with the one derived in Ref. [72]. The other value $c_{r 3}$ is the propagation speed of scalar-field perturbation $\delta \phi$. The presence of matter affects $c_{r 3}$ through the term proportional to $\rho+P$ in the denominator of Eq. (5.30). The radial Laplacian stabilities of $\psi$ and $\delta \phi$ are ensured under the two conditions $c_{r 2}^{2} \geq 0$ and $c_{r 3}^{2} \geq 0$. We note that the radial speed squares $(5.29)$ and (5.30) are different from those derived in Ref. [79]. In Ref. [79], the propagations of $\psi$ and $\delta \phi$ are affected by inappropriate treatment for the integration of the Schutz-Sorkin action. Now, we corrected the values of $c_{r 2}^{2}$ and $c_{r 3}^{2}$ together with the matter propagation speed squared $c_{r 1}^{2}$.

Since the matter perturbation $\delta \rho$ is decoupled from $\psi$ and $\delta \phi$ for high frequency modes, the no-ghost condition for $\delta \rho$ corresponds to the standard weak energy condition, i.e.,

$$
\rho+P>0
$$

under which $K_{11}$ is positive. The quantities associated with the no ghost conditions of other two dynamical perturbations are given by

$$
\begin{aligned}
K_{22} & =\frac{r^{2} \mathcal{H}}{4 \sqrt{f h}}, \\
K_{22} K_{33}-K_{23} K_{32} & =\frac{r}{8 f h^{2} \phi^{\prime 2} \mu}\left[\left(2 \mathcal{P}_{1}-\mathcal{F}\right) h \mu^{2}-2 \mathcal{H}^{2} r^{4}(\rho+P)\right] .
\end{aligned}
$$

The stability of odd-parity perturbations requires that $\mathcal{H}>0$, under which $K_{22}$ is positive. Since there are freedoms to multiply any positive or negative quantities to the third equation of (5.16), the sign of $K_{22} K_{33}-K_{23} K_{32}$ does not necessarily fix the no-ghost condition. Without the perfect fluid, however, we know that the ghost is absent for $2 \mathcal{P}_{1}-\mathcal{F}>0[72]$, whose combination appears in Eq. (5.33). Provided that the terms inside the square bracket of Eq. (5.33) are positive, i.e.,

$$
\mathcal{K} \equiv\left(2 \mathcal{P}_{1}-\mathcal{F}\right) h \mu^{2}-2 \mathcal{H}^{2} r^{4}(\rho+P)>0
$$

we recover the no-ghost condition without matter. Then the inequality (5.34) can be regarded as the no-ghost condition in the presence of matter. Under the condition (5.34) the denominator of Eq. (5.30) is positive, so the inequality $c_{r 3}^{2} \geq 0$ requires that the numerator of Eq. (5.30) is nonnegative. In summary, the absence of ghosts in the even-parity sector imposes the two additional conditions (5.31) and (5.34).

\section{Angular propagation}

Let us proceed to the derivation of propagation speeds along the angular direction. In doing so, we substitute the solution of the form $\overrightarrow{\mathcal{X}}=\overrightarrow{\mathcal{X}}_{0} e^{i(\omega t-l \theta)}$ into the perturbation equations (5.16). In the limits of large $\omega$ and $l$, the dispersion relation is given by $\operatorname{det}\left|\omega^{2} \boldsymbol{K}-\boldsymbol{M}\right|=0$. The angular propagation speed $\hat{c}_{\Omega}=r \mathrm{~d} \theta / \mathrm{d} t \operatorname{satisfies} \omega^{2}=\hat{c}_{\Omega}^{2} L / r^{2}$ in the limit $l \rightarrow \infty$. The speed $c_{\Omega}$ measured in proper time $\tau$ is $c_{\Omega}=r \mathrm{~d} \theta / \mathrm{d} \tau=\hat{c}_{\Omega} / \sqrt{f}$, so that $\omega^{2}=c_{\Omega}^{2} f L / r^{2}$. Then, we can derive $c_{\Omega}^{2}$ by solving

$$
\operatorname{det}\left|f L c_{\Omega}^{2} \boldsymbol{K}-r^{2} \boldsymbol{M}\right|=0
$$

Since the components $M_{21}$ and $M_{31}$ of matrix $\boldsymbol{M}$ do not vanish, the matter propagation speed does not apparently decouple from other speeds of propagation. However, we will see that the former eventually reduces to $c_{m}^{2}$ after the following manipulation. Expanding the matrix components of $M$ in the limit $L \rightarrow \infty$, the leading-order components of $M_{1 i}, M_{2 i}$ (where $i=1,2,3$ ) have the dependence linearly in $L$, whereas $M_{3 i}$ 's have the leading-order terms proportional to $L^{2}$. Substituting the expanded components of $\boldsymbol{M}$ into Eq. (5.35), the leading-order terms are in proportion to $L^{4}$. However, they vanish identically after using the relations among the coefficients given in Appendix A together 
with Eqs. (5.25) and (5.27). Then, we need to pick up the next-to leading-order terms proportional to $L^{3}$. Then, the dispersion relation (5.35) gives the following equation

$$
\left(c_{\Omega}^{2}-c_{m}^{2}\right)\left(c_{\Omega}^{4}+2 B_{1} c_{\Omega}^{2}+B_{2}\right)=0,
$$

where

$$
\begin{aligned}
& B_{1}=\frac{a_{4} \sqrt{h} r^{3}\left[4 h\left(\phi^{\prime} a_{1}+2 r a_{4}\right) \beta_{1}+\beta_{2}-4 \phi^{\prime} a_{1} \beta_{3}+2 f r a_{4} \mathcal{G}(\rho+P)\right]-2 f h^{3 / 2} \mathcal{G}\left[2 r a_{4}\left(2 \mathcal{P}_{1}-\mathcal{F}\right)\left(\phi^{\prime} a_{1}+r a_{4}\right)+\phi^{\prime 2} a_{1}^{2} \mathcal{P}_{1}\right]}{4 \sqrt{f} a_{4}\left[\left(\phi^{\prime} a_{1}+2 r a_{4}\right)^{2}\left(2 \mathcal{P}_{1}-\mathcal{F}\right) h-2 r^{4} a_{4}^{2}(\rho+P)\right]} \\
& B_{2}=-r^{2} \frac{r^{2} h \beta_{1}\left[2 f h \mathcal{F} \mathcal{G}\left(\phi^{\prime} a_{1}+2 r a_{4}\right)+r^{2} \beta_{2}\right]-r^{4} \beta_{2} \beta_{3}-f h \mathcal{F} \mathcal{G}\left(\phi^{\prime} f h \mathcal{F} \mathcal{G} a_{1}+4 r^{3} a_{4} \beta_{3}\right)}{f \mathcal{F} \phi^{\prime} a_{1}\left[\left(\phi^{\prime} a_{1}+2 r a_{4}\right)^{2}\left(2 \mathcal{P}_{1}-\mathcal{F}\right) h-2 r^{4} a_{4}^{2}(\rho+P)\right]}
\end{aligned}
$$

with

$$
\begin{aligned}
\beta_{1}= & \phi^{\prime 2} a_{4} e_{4}-2 \phi^{\prime} c_{4} a_{4}^{\prime}+\left[\left(\frac{f^{\prime}}{f}+\frac{h^{\prime}}{h}-\frac{2}{r}\right) a_{4}+\frac{\sqrt{f h} \mathcal{F}}{r}\right] \phi^{\prime} c_{4}+\frac{f \mathcal{F} \mathcal{G}}{2 r^{2}}, \\
\beta_{2}= & {\left[\frac{\sqrt{f h} \mathcal{F}}{r^{2}}\left(2 h r \phi^{\prime 2} c_{4}+\frac{r f^{\prime} \phi^{\prime} a_{4}}{f}-\sqrt{f h} \phi^{\prime} \mathcal{G}\right)-\frac{2 \sqrt{f h} \phi^{\prime} a_{4} \mathcal{G}}{r}\left(\frac{\mathcal{G}^{\prime}}{\mathcal{G}}-\frac{a_{4}^{\prime}}{a_{4}}+\frac{f^{\prime}}{f}+\frac{1}{2} \frac{h^{\prime}}{h}-\frac{1}{r}\right)\right] a_{1}-\frac{4 \mathcal{F} \mathcal{G} f h a_{4}}{r}, } \\
\beta_{3}= & \left(h c_{4}^{\prime}-\frac{d_{3}}{2}+\frac{1}{2} h^{\prime} c_{4}\right) \phi^{\prime} a_{4}+\left(\frac{h^{\prime}}{2 h}-\frac{1}{r}+\frac{f^{\prime}}{2 f}-\frac{a_{4}^{\prime}}{a_{4}}\right)\left(\frac{a_{4} f^{\prime}}{2 f}+2 h \phi^{\prime} c_{4}+\frac{\sqrt{f h} \mathcal{G}}{2 r}\right) a_{4} \\
& +\frac{\sqrt{f h} \mathcal{F}}{4 r}\left(\frac{f^{\prime}}{f} a_{4}+2 h \phi^{\prime} c_{4}+\frac{3 \sqrt{f h} \mathcal{G}}{r}\right) .
\end{aligned}
$$

In deriving these coefficients, we replaced $f^{\prime \prime}$ with $a_{4}^{\prime}$ on account of Eq. (5.27).

One of the solutions to Eq. (5.36) corresponds to the matter propagation speed squared, i.e.,

$$
c_{\Omega 1}^{2}=c_{m}^{2} .
$$

Hence, as in the case of radial mode, the propagation speed of matter along the angular direction is decoupled from those of other perturbations. The other two propagation speeds $c_{\Omega \pm}$ are given by

$$
c_{\Omega \pm}^{2}=-B_{1} \pm \sqrt{B_{1}^{2}-B_{2}} .
$$

Since there exist terms proportional to $\rho+P$ in the denominators of $B_{1}$ and $B_{2}$, the angular propagations of $\psi$ and $\delta \phi$ are generally affected by the presence of matter. The angular Laplacian instabilities of $\psi$ and $\delta \phi$ are absent under the two conditions $c_{\Omega+}^{2} \geq 0$ and $c_{\Omega-}^{2} \geq 0$.

$$
\text { B. } l=0
$$

For the monopole mode $(l=0)$, the terms containing $h_{0}, h_{1}$, and $G$ in the second-order Lagrangian (4.19) vanish identically. We choose the gauge $K=0$ to fix the residual gauge degree of freedom. We can exploit the perturbation Eqs. (5.9)-(5.15) by setting $h_{1}$ and its derivatives 0 , with $L=0$. To eliminate nondynamical perturbations, we first solve Eqs. (5.13) and (5.15) for $H_{0}^{\prime}$ and $H_{0}$ respectively. We take the $r$ derivative of Eq. (5.13) and solve it for $\ddot{\delta \phi}$. Substituting these relations into Eqs. (5.9)-(5.11), three perturbations $\overrightarrow{\mathcal{X}}={ }^{t}(\delta \rho, \psi, \delta \phi)$ obey equation of the form (5.16).

Let us consider the radial propagation of dynamical perturbations for high frequency modes. The dispersion relation is given by Eq. (5.18), with the same values of $K_{21}, K_{31}, G_{21}, G_{31}$ and $K_{11}, G_{11}$ as those in Eqs. (5.19), (5.20) and (5.22). Then, $\delta \rho$ propagates with the radial speed squared $c_{r 1}^{2}=c_{m}^{2}$. An important difference from the $l \geq 2$ case is that the matrix components $G_{22}$ and $G_{32}$ vanish. Then, one of the solutions to Eq. (5.18) is $c_{r 2}^{2}=0$, so that $\psi$ does not propagate. The other solution associated with the propagation speed squared of $\delta \phi$ is

$$
c_{r 3}^{2}=\frac{K_{32} G_{23}-K_{22} G_{33}}{f h\left(K_{22} K_{33}-K_{23} K_{32}\right)}=\frac{4 \phi^{\prime}\left[8 r^{2} f h a_{4} c_{4}\left(\phi^{\prime} a_{1}+r a_{4}\right)-a_{1}^{2} f^{3 / 2} \phi^{\prime} \mathcal{G} \sqrt{h}+2\left(a_{1} f^{\prime}+2 c_{2} f\right) a_{4}^{2} r^{2}\right]}{f^{5 / 2} \sqrt{h}\left[\left(2 \mathcal{P}_{1}-\mathcal{F}\right) h \mu^{2}-2 \mathcal{H}^{2} r^{4}(\rho+P)\right]},
$$

which is identical to Eq. (5.30). Term $K_{22} K_{33}-K_{23} K_{32}$, which is associated with the no-ghost condition, is also the same as Eq. (5.33). In summary, for $l=0$, the propagations of two dynamical perturbations $\delta \rho$ and $\delta \phi$ along the radial direction occur in the same manner as that for $l \geq 2$, with no propagation of the gravitational degree of freedom. 


\section{C. $l=1$}

Let us proceed to the dipole mode $(l=1)$. On using two relations $g_{5}=-2 g_{3}$ and $g_{6}=-2 g_{4}$ with $L=2$ in Eq. (4.19), terms containing the dependence of $G$ and $K$ always appear as the derivatives of $G-K$. We choose the gauges $h_{0}=0, K=0$, and $G=0$, but this does not completely fix the gauge degrees of freedom. Then, we impose the gauge condition $\delta \phi=0$ further. For $L=2$, the left hand-side of Eq. (4.36) vanishes identically, so we cannot exploit Eq. (5.15) to eliminate $H_{0}$. Instead, we resort to Eq. (4.31) together with Eq. (4.28). Taking the time derivative of Eq. (4.31) and using Eqs. (4.26)-(4.28), it follows that

$$
\frac{1}{f}\left[r^{2}(\rho+P) \sqrt{f h}-2 a_{4}\right] \dot{H}_{1}+\frac{2 a_{3}}{f} \ddot{\psi}-\frac{2 a_{4}}{f} \ddot{h}_{1}+\frac{\sqrt{h}}{2 f} r^{2}(\rho+P)\left(f^{\prime} \dot{v}-2 f \dot{v}^{\prime}\right)=0 .
$$

From Eq. (5.8), we can express $\dot{v}$ and $\dot{v}^{\prime}$ with respect to $\delta \rho, H_{0}, \delta \rho^{\prime}$, and $H_{0}^{\prime}$.

We solve Eqs. (5.12), (5.13), (5.14), and (5.45) for $h_{1}, \dot{H}_{1}, H_{0}$, and $\ddot{h}_{1}$, respectively. After taking the $r$ derivative of Eqs. (5.12) and (5.13), we also express $h_{1}^{\prime}$ and $H_{0}^{\prime \prime}$ with respect to other perturbations. Substituting all these relations into Eqs. (5.9) and (5.10), two dynamical perturbations $\overrightarrow{\mathcal{X}}={ }^{t}(\delta \rho, \psi)$ obey equation of the form (5.16) with $2 \times 2$ matrices $\boldsymbol{K}, \boldsymbol{G}, \boldsymbol{Q}, \boldsymbol{M}$. For high-frequency modes the dispersion relation along the radial direction is given by Eq. (5.18). Since $K_{21}=0$ and $G_{21}=0$ with the same values of $K_{11}$ and $G_{11}$ as those in Eq. (5.22), $\delta \rho$ propagates with the sound speed squared $c_{r 1}^{2}=c_{m}^{2}$. The propagation speed squared of $\psi$ is

$$
c_{r 3}^{2}=-\frac{G_{22}}{f h K_{22}}=\frac{4 \phi^{\prime}\left[8 r^{2} f h a_{4} c_{4}\left(\phi^{\prime} a_{1}+r a_{4}\right)-a_{1}^{2} f^{3 / 2} \phi^{\prime} \mathcal{G} \sqrt{h}+2\left(a_{1} f^{\prime}+2 c_{2} f\right) a_{4}^{2} r^{2}\right]}{f^{5 / 2} \sqrt{h}\left[\left(2 \mathcal{P}_{1}-\mathcal{F}\right) h \mu^{2}-2 \mathcal{H}^{2} r^{4}(\rho+P)\right]} .
$$

This is equivalent to Eq. (5.30), i.e., the sound speed squared of $\delta \phi$. The matrix component $K_{22}$ is also proportional to $\mathcal{K}$ given by Eq. (5.34). Thus, for $l=1$, the matter and scalar-field perturbations propagate with the same forms of radial sound speeds as those derived for $l=0$. These results are consistent with those obtained in Ref. [72] without the perfect fluid.

\section{CONCRETE THEORIES}

Let us apply the stability conditions derived in Secs. III and V to two concrete theories in which hairy relativistic star solutions are known to exist. The first class is the case in which the nonminimal coupling $G_{4}(\phi) R$ is present. The second class is a derivative coupling with the Einstein tensor of the form $\phi G_{\mu \nu} \nabla^{\mu} \nabla^{\nu} \phi$.

\section{A. Nonminimally coupled theories}

The theories of spontaneous scalarization, $f(R)$ gravity, and Brans-Dicke theories can be accommodated by the action

$$
\mathcal{S}=\int \mathrm{d}^{4} x \sqrt{-g}\left[G_{4}(\phi) R+G_{2}(\phi, X)\right]+\mathcal{S}_{m}\left(g_{\mu \nu}, \Psi_{m}\right) .
$$

In the odd-parity sector, the stability conditions are ensured for

$$
\mathcal{H}=\mathcal{F}=\mathcal{G}=2 G_{4}>0 .
$$

The radial and angular propagation speed squares for the odd-mode gravitational perturbation $\chi$ are

$$
c_{r}^{2}=1, \quad c_{\Omega}^{2}=1 .
$$

In the even-parity sector, the matter perturbation $\delta \rho$ propagates with the radial and angular speed squares given by

$$
c_{r 1}^{2}=c_{m}^{2}, \quad c_{\Omega 1}^{2}=c_{m}^{2} .
$$

Using the background Eqs. (2.20) and (2.21), the radial propagation speed squares (5.29) and (5.30) of the perturba-

tions $\psi$ and $\delta \phi$ reduce, respectively, to

$$
\begin{aligned}
& c_{r 2}^{2}=1, \\
& c_{r 3}^{2}=\frac{\kappa+2 X G_{2, X X} G_{4}}{\kappa},
\end{aligned}
$$


where

$$
\kappa \equiv G_{2, X} G_{4}+3 G_{4, \phi}^{2}
$$

The no-ghost condition (5.34) translates to $\mathcal{K}=8 \phi^{\prime 2} h r^{4} G_{4} \kappa>0$. Under the condition (6.2), this is satisfied for

$$
\kappa>0
$$

Since $B_{1}=-1$ and $B_{2}=1$ for the theories under consideration, the angular propagation speed squares (5.43) in the even-parity sector reduce to

$$
c_{\Omega+}^{2}=c_{\Omega-}^{2}=1
$$

These results show that, apart from $c_{r 3}^{2}$, all the other propagation speeds are equivalent to that of light. In the following, we consider more specific theories that belong to the action (6.1).

\section{Theories of spontaneous scalarization}

In the Jordan frame, theories of spontaneous scalarization are given by the functions [79, 96]

$$
G_{4}(\phi)=\frac{M_{\mathrm{pl}}^{2}}{2} F(\phi), \quad G_{2}(\phi, X)=\left(1-\frac{3 M_{\mathrm{pl}}^{2} F_{, \phi}^{2}}{2 F^{2}}\right) F(\phi) X,
$$

where $M_{\mathrm{pl}}$ is the reduced Planck mass. For the realization of spontaneous scalarization, the nonminimal coupling $F(\phi)$ contains even power-law functions of $\phi$. Since $G_{2, X X}=0$, it follows that $c_{r 3}^{2}=1$. The no-ghost condition (6.8) yields

$$
\kappa=\frac{M_{\mathrm{pl}}^{2}}{2} F^{2}(\phi)>0,
$$

which is automatically satisfied. Hence the odd- and even-parity stabilities of scalarized solutions in theories of spontaneous scalarization are ensured under the condition (6.2), i.e.,

$$
F(\phi)>0
$$

besides the condition $\rho+P>0$ in the matter sector. This main result is the same as that found in Ref. [79], in spite of different values $c_{r 1}^{2}, c_{r 2}^{2}, c_{r 3}^{2}$, and $c_{\Omega+}^{2}$.

\section{Brans-Dicke theories}

Brans-Dicke theories with a potential $V(\phi)$ are given by the functions [35]

$$
G_{4}(\phi)=\frac{1}{2} \phi, \quad G_{2}(\phi, X)=\frac{\omega_{\mathrm{BD}}}{\phi} X-V(\phi)
$$

where $\omega_{\mathrm{BD}}$ is a constant, and we used the unit $M_{\mathrm{pl}}=1$. Note that $f(R)$ gravity corresponds to the special case $\omega_{\mathrm{BD}}=0$. Since $G_{2, X X}=0$, we have $c_{r 3}^{2}=1$. The stability conditions (6.2) and (6.8) reduce, respectively, to

$$
2 G_{4}=\phi>0, \quad \kappa=\frac{1}{4}\left(2 \omega_{\mathrm{BD}}+3\right)>0 .
$$

Under these conditions, there are neither ghost nor Laplacian instabilities for hairy relativistic star solutions in Brans-Dicke theories. 


\section{B. Derivative coupling to Einstein tensor}

Theories of a derivative coupling to the Einstein tensor are given by the action [68, 69]

$$
\mathcal{S}=\int \mathrm{d}^{4} x \sqrt{-g}\left[\frac{1}{16 \pi G_{\mathrm{N}}} R-\frac{1}{2} \eta \phi G_{\mu \nu} \nabla^{\mu} \nabla^{\nu} \phi\right]+\mathcal{S}_{m},
$$

where $G_{\mathrm{N}}=\left(8 \pi M_{\mathrm{pl}}^{2}\right)^{-1}$ is Newton gravitational coupling, and $\eta$ is a constant. The action (6.15) corresponds to the functions $G_{4}=1 /\left(16 \pi G_{\mathrm{N}}\right)$ and $G_{5}(\phi)=-\eta \phi / 2$. From Eq. (2.28), the background scalar field obeys

$$
\eta \sqrt{\frac{h}{f}} \phi^{\prime}\left[f(1-h)-r f^{\prime} h\right]=0
$$

where we imposed the boundary conditions $f \rightarrow 1, h \rightarrow 1$, and $\phi^{\prime} \rightarrow 0$ at spatial infinity. Then, there is a nontrivial branch satisfying

$$
f^{\prime}=\frac{f(1-h)}{h r} .
$$

Substituting Eq. (6.17) into Eq. (2.21), we obtain

$$
\eta h \phi^{\prime 2}=\operatorname{Pr}^{2}
$$

This means that the nontrivial branch (6.17) corresponds to a solution where $\phi^{\prime}$ does not vanish only inside the star $(P>0)$. Substituting Eq. (6.18) and its $r$ derivative as well as Eq. (2.24) into Eq. (2.20), it follows that

$$
h^{\prime}=\frac{1-h-4 \pi G_{\mathrm{N}} r^{2}[(1+h) \rho+6 h P]}{r\left(1+4 \pi G_{\mathrm{N}} r^{2} P\right)} .
$$

Solving Eqs. (2.24), (6.17), (6.18), and (6.19) for a given NS equation of state, we obtain a hairy solution where the metric components are modified from those in GR inside the star [68,69]. The stabilities of such hairy NS solutions were studied in Ref. [80], but the treatment of how to integrate out the Schutz-Sorkin action was not appropriate as in Ref. [79]. In the following, we will reconsider the stabilities of hairy NS solutions satisfying the relation (6.18).

The stabilities of odd-parity perturbations require that

$$
\mathcal{H}=\mathcal{G}=\frac{1}{8 \pi G_{\mathrm{N}}}+\frac{1}{2} \operatorname{Pr}^{2}>0, \quad \mathcal{F}=\frac{1}{8 \pi G_{\mathrm{N}}}-\frac{1}{2} \operatorname{Pr}^{2}>0 .
$$

For $P>0$ the former is trivially satisfied, while the latter gives $\operatorname{Pr}^{2}<1 /\left(4 \pi G_{\mathrm{N}}\right)$.

Under the conditions $(6.20)$, the radial propagation speed squared $c_{r 2}^{2}=\mathcal{G} / \mathcal{F}$ in the even-parity sector is positive. The other value (5.30) reduces to

$$
c_{r 3}^{2}=\frac{r^{6} P^{2}\left(1+4 \pi G_{\mathrm{N}} r^{2} P\right)}{2 \pi G_{\mathrm{N}} \mathcal{K}},
$$

where

$$
\mathcal{K}=\frac{r^{6} P}{8 \pi G_{\mathrm{N}}}\left[(3 h-1) \rho\left(1+4 \pi G_{\mathrm{N}} r^{2} P\right)+4(27 h-1) \pi G_{\mathrm{N}} r^{2} P^{2}+(19 h-1) P\right]
$$

The absence of ghosts requires that $\mathcal{K}>0$, under which $c_{r 3}^{2} \geq 0$. Expanding Eq. (6.22) around $P=0$, it follows that

$$
\mathcal{K}=\frac{r^{6}(3 h-1) \rho}{8 \pi G_{\mathrm{N}}} P+\mathcal{O}\left(P^{2}\right)
$$

Around the surface of NSs, the pressure $P$ approaches 0 and hence the dominant contribution to $\mathcal{K}$ is the first term on the right hand-side of Eq. (6.23). Then, we require the condition

$$
h>\frac{1}{3} \text {. }
$$


The explicit forms of $c_{\Omega \pm}^{2}$ are complicated, so we do not write their expressions here. However, the expansion of Eq. (5.43) around $P=0$ leads to

$$
\begin{aligned}
& c_{\Omega+}^{2}=1+\mathcal{O}\left(P^{2}\right), \\
& c_{\Omega-}^{2}=-\frac{3\left(1-h^{2}\right)}{4 h(3 h-1)}+\mathcal{O}(P) .
\end{aligned}
$$

The metric component $h$ is related to a positive mass function $M(r)$ as $h(r)=1-2 G M(r) / r$, and hence $h<1$. Under the no-ghost condition $h>1 / 3$ the leading-order contribution to Eq. (6.26) is negative, so that $c_{\Omega-}^{2}<0$. Then, the angular Laplacian instability around the surface of star is inevitable for the hairy NS solution discussed above. This fact was first recognized in Ref. [80], but it was based on the values of $c_{r 1}^{2}, c_{r 2}^{2}, c_{r 3}^{2}, \mathcal{K}, c_{\Omega+}^{2}$, and $c_{\Omega-}^{2}$ derived by inappropriate treatment for the integration of the Schutz-Sorkin action. In spite of this difference, the leading-order term in Eq. (6.26) is exactly the same as that obtained in Ref. [80], together with the no-ghost condition $h>1 / 3$. Hence the angular Laplacian instability in the even-parity sector does not allow for the existence of hairy NS solutions in derivative coupling theories given by the action (6.15).

\section{CONCLUSIONS}

In this paper, we studied relativistic star perturbations on a static and spherically symmetric background in Horndeski theories given by the action (2.1). We implemented a perfect fluid inside the star as a form of the Schutz-Sorkin action (2.3). We performed the analysis for the full Horndeski Lagrangian (2.2), while the previous studies [79, 80] restricted to a subclass of Horndeski theories. Moreover, we decomposed the perturbations into odd- and even-parity modes arising from gravity, scalar-field, and matter sectors, and derived the full second-order actions without fixing particular gauges. In our formulation, we can choose any convenient gauges depending on the problems at hand. To our knowledge, this gauge-ready formulation was not addressed before even for a sub-class of Horndeski theories.

We first showed that the second-order action of odd-parity perturbations is of the form (3.12). The reduced Lagrangian (3.13) contains three metric perturbations $W, Q, U$ and perturbations $\delta j, \delta \mathcal{A}, \delta \mathcal{B}$ arising from the perfect fluid. Choosing the gauge $U=0$ and using the perturbation equations in the matter sector, we showed that the dynamical perturbation $\chi=\dot{W}-Q^{\prime}+2 Q / r$ obeys Eq. (3.33). The field $\chi$ is coupled to a nondynamical fluid-velocity perturbation $\delta j$ satisfying $\dot{\delta j}=0$, whose property is consistent with the corresponding equation in GR [95]. For the modes of high frequencies and large multipoles, the stability conditions in the odd-parity sector are not affected by the presence of matter.

The second-order action of even-parity perturbations is given by Eq. (4.18), where the associated Lagrangian (4.19) contains sixteen perturbed variables. We derived the full linear perturbation equations of motion without fixing gauges. In Sec. V, we chose the gauge conditions $h_{0}=0, G=0$, and $K=0$ for the multipoles $l \geq 2$ and studied the propagation of even-parity modes. Introducing a dynamical perturbation $\psi$ arising from the gravity sector and eliminating all nondynamical variables, the dynamical perturbations $\overrightarrow{\mathcal{X}}={ }^{t}(\delta \rho, \psi, \delta \phi)$ satisfy closed-form equations of the form (5.16). Among them, the matter perturbation is decoupled from others for high-frequency modes, so that the radial propagation speed squared of $\delta \rho$ is given by $c_{r 1}^{2}=c_{m}^{2}$. We also obtained the propagation speed squares of $\psi$ and $\delta \phi$ in the forms (5.29) and (5.30), respectively. While $c_{r 2}^{2}$ coincides with the one derived in Ref. [72], $c_{r 3}^{2}$ is subject to modification by the presence of matter. We also showed that, for both $l=0$ and $l=1$, the radial propagations of the perfect fluid and scalar field occur in the same manner as those for $l \geq 2$, with no propagation of the gravitational perturbation.

For large multipoles $l(\gg 1)$, we derived the angular propagation speeds of even-parity perturbations by solving the dispersion relation (5.35). Like the radial mode, $\delta \rho$ is decoupled from $\psi$ and $\delta \phi$, so that the matter perturbation propagates with the speed squared $c_{\Omega 1}^{2}=c_{m}^{2}$ in the angular direction. We also obtained the angular speed squares of $\psi$ and $\delta \phi$ in the forms (5.43), where $B_{1}$ and $B_{2}$ are given by Eqs. (5.37) and (5.38), respectively. These values of $c_{\Omega \pm}^{2}$ were not derived in the literature even in the absence of matter. Given that there are some modified gravity (vector-tensor) theories in which hairy BH solutions are subject to Laplacian instabilities along the angular direction $[94,97]$, our general expressions of $c_{\Omega \pm}^{2}$ will be useful to select stable NS and BH solutions in Horndeski theories.

In Sec. VI, we applied our stability conditions to hairy NS solutions in several sub-classes of Horndeski theories. Note that similar investigations were performed in Refs. [79, 80], but the prescription of how to integrate out the Schutz-Sorkin action was not appropriate in these works. In Appendix B, we clarified this issue by deriving an effective matter action of the form (A.13). The analyses of Refs. [79, 80] led to some different propagation speeds of evenparity perturbations in comparison to those in the current paper. On using the correct radial and angular propagation speeds, the main stability conditions discussed in Refs. [79, 80] are hardly subject to modifications. For example, scalarized solutions in theories of spontaneous scalarization are stable under the condition $G_{4}(\phi)>0$. Hairy solutions 
in theories of the derivative coupling to the Einstein tensor are subject to the angular Laplacian instability around the surface of NSs. In particular, the latter fact shows the importance of scrutinizing the stabilities of NSs and BHs against both odd- and even-parity perturbations before computing quasi-normal modes of odd-parity perturbations alone [98, 99].

It will be of interest to judge the stabilities of hairy NS and BH solutions by using our general conditions derived in this paper. The applications to the tidal deformation of a NS-NS binary system and to the calculations of NS and $\mathrm{BH}$ quasi-normal frequencies will be the next important steps to probe the physics of strong gravity regimes through the observations of GWs.

\section{Acknowledgements}

We thank Rampei Kimura, Kei-ichi Maeda, and Seiga Sato for useful discussions. RK is supported by the Grantin-Aid for Young Scientists B of the JSPS No. 20K14471. ST is supported by the Grant-in-Aid for Scientific Research Fund of the JSPS No. 19K03854.

\section{Appendix A: Coefficients in the second-order action of even-parity perturbations}

The coefficients of the reduced Lagrangian (4.19) of even-parity perturbations are

$$
\begin{aligned}
& a_{1}=\sqrt{f h}\left[\left\{G_{4, \phi}+\frac{1}{2} h\left(G_{3, X}-2 G_{4, \phi X}\right) \phi^{\prime 2}\right\} r^{2}+2 h \phi^{\prime}\left\{G_{4, X}-G_{5, \phi}-\frac{1}{2} h\left(2 G_{4, X X}-G_{5, \phi X}\right) \phi^{\prime 2}\right\} r\right. \\
& \left.+\frac{1}{2} G_{5, X X} h^{3} \phi^{\prime 4}-\frac{1}{2} G_{5, X} h(3 h-1) \phi^{\prime 2}\right] \text {, } \\
& a_{2}=\sqrt{f h}\left(\frac{a_{1}}{\sqrt{f h}}\right)^{\prime}-\left(\frac{\phi^{\prime \prime}}{\phi^{\prime}}-\frac{1}{2} \frac{f^{\prime}}{f}\right) a_{1}+\frac{r}{\phi^{\prime}}\left(\frac{f^{\prime}}{f}-\frac{h^{\prime}}{h}\right) a_{4}-\frac{1}{2} \frac{\sqrt{f} r^{2}(\rho+P)}{\sqrt{h} \phi^{\prime}}, \\
& a_{3}=-\frac{1}{2} \phi^{\prime} a_{1}-r a_{4}, \quad a_{4}=\frac{\sqrt{f h}}{2} \mathcal{H}, \quad a_{5}=a_{2}^{\prime}-a_{1}^{\prime \prime}, \quad a_{6}=-\frac{\sqrt{f}}{2 \sqrt{h} \phi^{\prime}}\left(\mathcal{H}^{\prime}+\frac{\mathcal{H}}{r}-\frac{\mathcal{F}}{r}\right), \\
& a_{7}=a_{3}^{\prime}+\frac{1}{4} \frac{\sqrt{f} r^{2}(\rho+P)}{\sqrt{h}}, \quad a_{8}=-\frac{1}{2} \frac{a_{4}}{h}, \quad a_{9}=a_{4}^{\prime}+\left(\frac{1}{r}-\frac{1}{2} \frac{f^{\prime}}{f}\right) a_{4}, \quad a_{10}=\frac{1}{2} \frac{r^{2} \sqrt{f}}{\sqrt{h}}, \\
& b_{1}=\frac{1}{2 f} a_{4}, \quad \tilde{b}_{1}=-\frac{h r^{2}}{f} f_{1}, \quad b_{2}=-\frac{2}{f} a_{1}, \quad b_{3}=-\frac{2}{f}\left(a_{2}-a_{1}^{\prime}\right), \quad b_{4}=-\frac{2}{f} a_{3}, \quad b_{5}=-2 b_{1}, \\
& c_{1}=-\frac{1}{f h} a_{1} \\
& c_{2}=\sqrt{f h}\left[\left\{\frac{1}{2 f}\left(-\frac{1}{2} h\left(3 G_{3, X}-8 G_{4, \phi X}\right) \phi^{\prime 2}+\frac{1}{2} h^{2}\left(G_{3, X X}-2 G_{4, \phi X X}\right) \phi^{\prime 4}-G_{4, \phi}\right) r^{2}\right.\right. \\
& -\frac{h \phi^{\prime}}{f}\left(\frac{1}{2} h^{2}\left(2 G_{4, X X X}-G_{5, \phi X X}\right) \phi^{\prime 4}-\frac{1}{2} h\left(12 G_{4, X X}-7 G_{5, \phi X}\right) \phi^{2}+3\left(G_{4, X}-G_{5, \phi}\right)\right) r \\
& \left.+\frac{h \phi^{\prime 2}}{4 f}\left(G_{5, X X X} h^{3} \phi^{\prime 4}-G_{5, X X} h(10 h-1) \phi^{2}+3 G_{5, X}(5 h-1)\right)\right\} f^{\prime} \\
& +\phi^{\prime}\left\{\frac{1}{2} G_{2, X}-G_{3, \phi}-\frac{1}{2} h\left(G_{2, X X}-G_{3, \phi X}\right) \phi^{\prime 2}\right\} r^{2} \\
& +2\left\{-\frac{1}{2} h\left(3 G_{3, X}-8 G_{4, \phi X}\right) \phi^{\prime 2}+\frac{1}{2} h^{2}\left(G_{3, X X}-2 G_{4, \phi X X}\right) \phi^{\prime 4}-G_{4, \phi}\right\} r \\
& \left.-\frac{1}{2} h^{3}\left(2 G_{4, X X X}-G_{5, \phi X X}\right) \phi^{\prime 5}+\frac{1}{2} h\left\{2(6 h-1) G_{4, X X}+(1-7 h) G_{5, \phi X}\right\} \phi^{\prime 3}-(3 h-1)\left(G_{4, X}-G_{5, \phi}\right) \phi^{\prime}\right] \text {, } \\
& c_{3}=-\frac{1}{2} \frac{\sqrt{f} r^{2}}{\sqrt{h}} \frac{\partial \mathcal{E}_{11}}{\partial \phi} \\
& c_{4}=\frac{1}{4} \frac{\sqrt{f}}{\sqrt{h}}\left[\frac{h \phi^{\prime}}{f}\left\{2 G_{4, X}-2 G_{5, \phi}-h\left(2 G_{4, X X}-G_{5, \phi X}\right) \phi^{\prime 2}-\frac{h \phi^{\prime}\left(3 G_{5, X}-G_{5, X X} \phi^{\prime 2} h\right)}{r}\right\} f^{\prime}\right.
\end{aligned}
$$




$$
\begin{aligned}
& \left.+4 G_{4, \phi}+2 h\left(G_{3, X}-2 G_{4, \phi X}\right) \phi^{\prime 2}+\frac{4 h\left(G_{4, X}-G_{5, \phi}\right) \phi^{\prime}-2 h^{2}\left(2 G_{4, X X}-G_{5, \phi X}\right) \phi^{\prime 3}}{r}\right], \\
& c_{5}=-h \phi^{\prime} c_{4}-\frac{1}{2} \frac{\sqrt{f h}}{r} \mathcal{G}-\frac{1}{2} \frac{f^{\prime}}{f} a_{4} \\
& c_{6}=\frac{1}{8} \frac{f^{\prime} \phi^{\prime}}{f} a_{1}+\frac{1}{2} \frac{f^{\prime} r}{f} a_{4}-\frac{1}{4} \phi^{\prime} c_{2}+\frac{1}{2} h \phi^{\prime} r c_{4}+\frac{1}{4} \sqrt{f h} \mathcal{G}, \\
& d_{1}=\frac{1}{2 f} a_{4}, \quad d_{2}=2 h c_{4}, \\
& d_{3}=-\frac{1}{r^{2}}\left(\frac{2 \phi^{\prime \prime}}{\phi^{\prime}}+\frac{h^{\prime}}{h}\right) a_{1}+\frac{2 f}{\left(f^{\prime} r-2 f\right) \phi^{\prime}}\left(\frac{2 \phi^{\prime \prime}}{h \phi^{\prime} r}+\frac{f^{\prime 2}}{f^{2}}-\frac{f^{\prime} h^{\prime}}{f h}-\frac{2 f^{\prime}}{f r}+\frac{2 h^{\prime}}{h r}+\frac{h^{\prime}}{h^{2} r}\right) a_{4} \\
& +\frac{f^{\prime} r-2 f}{f r} \frac{\partial a_{4}}{\partial \phi}+\frac{\sqrt{f}}{\phi^{\prime} \sqrt{h} r^{2}} \mathcal{F}-\frac{f^{3 / 2}}{\sqrt{h}\left(f^{\prime} r-2 f\right) \phi^{\prime}}\left(\frac{f^{\prime}}{f r}+\frac{2 \phi^{\prime \prime}}{\phi^{\prime} r}+\frac{h^{\prime}}{h r}-\frac{2}{r^{2}}\right) \mathcal{G}-\frac{\sqrt{f}(\rho+P)}{\phi^{\prime} \sqrt{h}}, \\
& d_{4}=\frac{1}{2} \frac{\sqrt{f h}}{r^{2}} \mathcal{G}, \\
& e_{1}=\frac{1}{\phi^{\prime} f h}\left[\left(\frac{f^{\prime}}{f}+\frac{1}{2} \frac{h^{\prime}}{h}\right) a_{1}-2 a_{1}^{\prime}+a_{2}-2 r h a_{6}\right] \text {, } \\
& e_{2}=-\frac{1}{2 \phi^{\prime}}\left(\frac{f^{\prime}}{f} a_{1}+2 c_{2}+4 h r c_{4}\right), \quad e_{3}=\frac{1}{4} \frac{\sqrt{f} r^{2}}{\sqrt{h}} \frac{\partial \mathcal{E}_{\phi}}{\partial \phi}, \\
& e_{4}=\frac{1}{\phi^{\prime}} c_{4}^{\prime}-\frac{1}{2} \frac{f^{\prime}}{f \phi^{\prime 2} h} a_{4}^{\prime}-\frac{1}{2} \frac{\sqrt{f}}{\phi^{\prime 2} \sqrt{h} r} \mathcal{G}^{\prime}+\frac{1}{h \phi^{\prime} r^{2}}\left(\frac{\phi^{\prime \prime}}{\phi^{\prime}}+\frac{1}{2} \frac{h^{\prime}}{h}\right) a_{1} \\
& +\frac{1}{4 h \phi^{\prime 2}}\left[\frac{\left(f^{\prime} r-6 f\right) f^{\prime}}{f^{2} r}+\frac{h^{\prime}\left(f^{\prime} r+4 f\right)}{h r f}-\frac{4 f\left(2 \phi^{\prime \prime} h+h^{\prime} \phi^{\prime}\right)}{\phi^{\prime} h^{2} r\left(f^{\prime} r-2 f\right)}\right] a_{4}+\frac{1}{2} \frac{h^{\prime}}{h \phi^{\prime}} c_{4}-\frac{1}{2} \frac{f^{\prime} r-2 f}{f h r \phi^{\prime}} \frac{\partial a_{4}}{\partial \phi} \\
& +\frac{1}{2} \frac{f^{\prime} h r-f}{r^{2} \sqrt{f} \phi^{\prime 2} h^{3 / 2}} \mathcal{F}+\frac{1}{2} \frac{\sqrt{f}}{r \phi^{\prime 2} h^{3 / 2}}\left[\frac{f\left(2 \phi^{\prime \prime} h+h^{\prime} \phi^{\prime}\right)}{h \phi^{\prime}\left(f^{\prime} r-2 f\right)}+\frac{1}{2} \frac{2 f-f^{\prime} h r}{f r}\right] \mathcal{G}+\frac{1}{2} \frac{\sqrt{f}(\rho+P)}{h^{3 / 2} \phi^{\prime 2}}, \\
& f_{1}=\frac{1}{2} \frac{\sqrt{f}(\rho+P)}{\sqrt{h}}, \quad f_{2}=-\frac{1}{2} \frac{r^{2} \sqrt{f} c_{m}^{2}}{\sqrt{h}(\rho+P)}, \quad f_{3}=\sqrt{h} n r^{2}, \quad f_{4}=\frac{n r^{2}}{\sqrt{h}(\rho+P)}, \quad f_{5}=\frac{1}{2} \frac{f_{3}}{h}, \\
& f_{6}=\sqrt{f} f_{3}, \quad f_{7}=\frac{1}{2} \frac{\sqrt{h} n r^{2}\left(c_{m}^{2}-1\right) f^{\prime}}{\sqrt{f} c_{m}^{2}}+\frac{1}{2} \frac{r^{2} \sqrt{f} h^{\prime} n}{\sqrt{h}}+2 r \sqrt{f h} n, \quad f_{8}=-\frac{\sqrt{f}}{h r^{2}} f_{3}, \\
& f_{9}=h r^{2} f_{1}, \quad f_{10}=-\sqrt{f} f_{3}, \quad f_{11}=-\frac{\sqrt{f}}{h r^{2}} f_{3}, \quad f_{12}=f_{3}, \quad f_{13}=-\frac{1}{h} f_{3}, \\
& g_{1}=\frac{r^{2}}{f} a_{4}, \quad g_{2}=-\frac{1}{2} r^{2} a_{4}, \quad g_{3}=-\frac{1}{8} \frac{r^{2}}{\sqrt{f h}} \mathcal{F}, \quad g_{4}=\frac{1}{8} r^{2} \sqrt{f h} \mathcal{G}, \quad g_{5}=-2 g_{3}, \\
& g_{6}=-2 g_{4}, \quad g_{7}=\frac{1}{2} \frac{r^{2}}{f h} a_{4}, \quad g_{8}=-\frac{r^{2}}{f} a_{6}, \quad g_{9}=-\frac{1}{2} \frac{r\left(f^{\prime} r-2 f\right)}{f^{2}} a_{4}, \quad g_{10}=-\frac{1}{2 h} f_{3}, \\
& g_{11}=\frac{1}{2 \sqrt{f h}} \mathcal{F}, \quad g_{12}=-h r^{2} c_{4}, \quad g_{13}=\frac{1}{4} \frac{r^{2} f^{\prime}}{f} a_{4}-\frac{1}{2} r^{2} a_{4}^{\prime}-\frac{3}{2} r a_{4}, \quad g_{14}=-\frac{1}{2} r^{2} c_{5}, \\
& g_{15}=-\frac{1}{2} r^{2} d_{3}, \quad g_{16}=-\frac{1}{2} \sqrt{f h} \mathcal{G}, \\
& k_{1}=-\frac{1}{4} \frac{\sqrt{f}}{\sqrt{h}} \mathcal{F}, \quad k_{2}=\frac{1}{4} \frac{\sqrt{f}}{\sqrt{h}} \mathcal{G}, \quad k_{3}=\frac{2 f \mathcal{G}^{\prime}+f^{\prime}(\mathcal{G}-\mathcal{F})}{4 \sqrt{f h} \phi^{\prime}}, \\
& m_{1}=\frac{1}{2 f} a_{4}, \quad m_{2}=-\frac{1}{f} a_{4}, \quad m_{3}=\frac{1}{f r} a_{4}^{\prime}+\frac{1}{f r^{2}}\left(1-\frac{r f^{\prime}}{f}\right) a_{4}-\frac{1}{2 \sqrt{f h} r^{2}} \mathcal{F}, \quad m_{4}=-\frac{1}{f h} a_{4}, \\
& m_{5}=\frac{2}{f r} a_{4}, \quad m_{6}=\frac{2}{f} a_{6}, \quad m_{7}=\frac{f^{\prime}}{f^{2}} a_{4}, \quad m_{8}=\frac{1}{h r^{2}} f_{3},
\end{aligned}
$$

where $\mathcal{H}, \mathcal{F}, \mathcal{G}, \mathcal{E}_{11}$, and $\mathcal{E}_{\phi}$ are given, respectively, by Eqs. (3.14), (3.15), (3.16), (2.21), and (2.28). 


\section{Appendix B: Effective matter Lagrangian in the even-parity sector}

We derive the effective matter Lagrangian for even-parity perturbations by integrating out most of the nondynamical variables. From Eq. (4.19), the Lagrangian containing the matter perturbations $v, \delta \mathcal{A}_{1}, \delta \mathcal{A}_{2}, \delta \mathcal{B}_{1}, \delta \mathcal{B}_{2}, \delta u_{r}, \delta \ell$, and $\delta \rho$ (with explicit coefficients) is given by

$$
\begin{aligned}
\mathcal{L}_{m}= & \frac{L \sqrt{f}(\rho+P)}{2 \sqrt{h}} v^{2}+\frac{n r^{2}}{\sqrt{h}}\left(h H_{1}-\delta \mathcal{B}_{1}\right) \delta \mathcal{A}_{1}+\frac{L n\left(h_{0}-\sqrt{f} v-r^{2} \delta \dot{\mathcal{B}}_{2}\right)}{\sqrt{h}} \delta \mathcal{A}_{2}+\frac{r^{2} \sqrt{f h}(\rho+P)}{2} \delta u_{r}^{2} \\
& -n r^{2} \sqrt{f h}\left(\delta \mathcal{A}_{1}+\delta \ell^{\prime}\right) \delta u_{r}+\frac{n r^{2}}{\sqrt{h}}\left[\frac{\dot{\delta} \rho}{\rho+P}+\dot{K}+\frac{\dot{H}_{2}}{2}-\frac{L \dot{G}}{2}+\frac{L}{r^{2}}\left(h_{0}-\sqrt{f} v\right)\right] \delta \ell+n r^{2} \sqrt{h} H_{1} \delta \ell^{\prime} \\
& -\frac{r^{2} \sqrt{f} c_{m}^{2}}{2 \sqrt{h}(\rho+P)} \delta \rho^{2}+\frac{r^{2} \sqrt{f}}{2 \sqrt{h}} H_{0} \delta \rho
\end{aligned}
$$

where we integrated the term $n r^{2} \sqrt{f h} \delta \ell \delta u_{r}^{\prime}$ by parts. As we mentioned at the end of Sec. III B, varying the Lagrangian (A.2) with respect to the perturbations with only its linear dependence and substituting the resulting equation into Eq. (A.2) does not generally give rise to a correct reduced Lagrangian. In Ref. [79], the authors varied the Lagrangian (A.2) with respect to $\delta \mathcal{B}_{1}$ and $\delta \mathcal{B}_{2}$, and plugged the resulting equations $\delta \dot{\mathcal{A}}_{1}=0$ and $\delta \dot{\mathcal{A}}_{2}=0$ into Eq. (A.2) in order to eliminate the terms proportional to $\delta \mathcal{B}_{1} \delta \mathcal{A}_{1}$ and $\delta \mathcal{B}_{2} \delta \mathcal{A}_{2}$. However, this process finally leads to an inconsistent value $c_{r 1}^{2}=0$ for the radial matter sound speed squared.

To obtain the reduced matter Lagrangian correctly, we first need to vary Eq. (A.2) with respect to perturbations containing the squared dependence in addition to the linear dependence. The quantity $v$ is such a variable, so the variation of Eq. (A.2) with respect to $v$ gives

$$
v=\frac{n}{\rho+P}\left(\delta \ell+\delta \mathcal{A}_{2}\right)
$$

which corresponds to Eq. (4.20). Now, we can substitute Eq. (A.3) into Eq. (A.2) to eliminate $v$. After this procedure the Lagrangian contains the terms proportional to $\delta \mathcal{A}_{2}^{2}$ as well as $\delta \mathcal{A}_{2}$, so the variation with respect to $\delta \mathcal{A}_{2}$ leads to

$$
\delta \mathcal{A}_{2}=-\delta \ell-\frac{\rho+P}{n \sqrt{f}}\left(r^{2} \dot{\delta \mathcal{B}_{2}}-h_{0}\right) .
$$

On using Eq. (A.3), it is clear that the relation (A.4) is consistent with (4.25). Substituting Eq. (A.4) into the Lagrangian (derived after the substitution of $v$ ) gives rise to the term proportional to $\dot{\delta \mathcal{B}_{2}^{2}}$, so the variation with respect to $\delta \mathcal{B}_{2}$ yields

$$
\dot{\delta \mathcal{B}_{2}}=\frac{h_{0}}{r^{2}}-\frac{n \sqrt{f}}{r^{2}(\rho+P)} \delta \ell+\mathcal{C}(r),
$$

where $\mathcal{C}(r)$ is a function of $r$. Then, from Eqs. (A.4) and (A.5), it follows that

$$
\delta \mathcal{A}_{2}=-\frac{r^{2}(\rho+P)}{n \sqrt{f}} \mathcal{C}(r) .
$$

The time derivative of this relation is consistent with Eq. (4.27). When we derived the second-order differential equation (5.9) of $\delta \rho$, we took the time derivative of Eq. (4.21) and eliminated the term $\dot{\delta} u_{r}$ by using Eq. (4.23). In this procedure, the $\delta \mathcal{A}_{2}$-dependent term vanishes due to the relation (4.27). Since the arbitrary function $\mathcal{C}(r)$ does not affect the dynamics of matter perturbations $\delta \rho$, we can set

$$
\mathcal{C}(r)=0 \quad \rightarrow \quad \delta \mathcal{A}_{2}=0
$$

without the loss of generality. Then, the term $\delta \mathcal{A}_{2}$ vanishes from the reduced Lagrangian.

The next step is to vary the reduced Lagrangian further with respect to $\delta u_{r}$. This leads to

$$
\delta u_{r}=\frac{n}{\rho+P}\left(\delta \ell^{\prime}+\delta \mathcal{A}_{1}\right)
$$


Taking the $r$ derivative of Eq. (4.20) and eliminating the term $\delta \ell^{\prime}$ from Eq. (A.8), we find that Eq. (A.8) is equivalent to Eq. (4.23). On using Eq. (A.8), the reduced Lagrangian possesses the term proportional to $\delta \mathcal{A}_{1}^{2}$, so the variation with respect to $\delta \mathcal{A}_{1}$ gives

$$
\delta \mathcal{A}_{1}=-\delta \ell^{\prime}+\frac{\rho+P}{n \sqrt{f} h}\left(h H_{1}-\dot{\delta \mathcal{B}_{1}}\right)
$$

which is consistent with Eq. (4.25) on account of Eq. (A.8). After eliminating $\delta \mathcal{A}_{1}$ from the Lagrangian, there are terms proportional to $\delta \mathcal{B}_{1}^{2}$, so the variation with respect to $\delta \mathcal{B}_{1}$ leads to

$$
\dot{\delta \mathcal{B}_{1}}=h H_{1}-\frac{n \sqrt{f} h}{\rho+P} \delta \ell^{\prime}+\mathcal{D}(r),
$$

where $\mathcal{D}(r)$ is a function of $r$. Substituting Eq. (A.10) into Eq. (A.9), we have

$$
\delta \mathcal{A}_{1}=-\frac{\rho+P}{n \sqrt{f} h} \mathcal{D}(r),
$$

whose time derivative is consistent with Eq. (4.26). The perturbation $\delta \mathcal{A}_{1}$ vanishes from the second-order differential equation (5.9) of $\delta \rho$, so we can set

$$
\mathcal{D}(r)=0 \quad \rightarrow \quad \delta \mathcal{A}_{1}=0
$$

On using Eq. (A.10) with Eq. (A.12), we end up with the reduced Lagrangian containing $\delta \rho, \delta \ell$, and their derivatives. From Eqs. (A.3) and (A.7) we have $\delta \ell=(\rho+P) v / n$, so $\delta \ell$ and $\delta \ell^{\prime}$ can be replaced with $v$ and $v^{\prime}$. Then, the reduced effective Lagrangian for the perfect fluid is given by

$$
\begin{aligned}
\mathcal{L}_{m}= & \frac{r^{2}}{\sqrt{h}}\left[v \dot{\delta} \rho+\frac{\sqrt{f}}{2} H_{0} \delta \rho-\frac{\sqrt{f}}{2(\rho+P)} c_{m}^{2} \delta \rho^{2}\right]-\frac{\rho+P}{8 f^{3 / 2} \sqrt{h}}\left[4 L f^{2} v^{2}+r^{2} h\left(2 f v^{\prime}-f^{\prime} v\right)^{2}\right] \\
& +\frac{\rho+P}{2 f \sqrt{h}}\left[2 L f h_{0} v-r^{2} f\left(L \dot{G}-\dot{H}_{2}-2 \dot{K}\right) v-r^{2} f^{\prime} h H_{1} v+2 r^{2} f h H_{1} v^{\prime}\right] .
\end{aligned}
$$

Varying (A.13) with respect to $v$ and $\delta \rho$, we obtain the perturbation equations same as Eqs. (4.21) and (4.22), respectively. For the purpose of deriving the perturbation equations, we can directly employ the reduced matter Lagrangian (A.13) besides the second-order Lagrangians of gravitational and scalar-field perturbations.

[1] B. P. Abbott et al., Phys. Rev. Lett. 116, 061102 (2016) [arXiv:1602.03837 [gr-qc]].

[2] B. P. Abbott et al., Phys. Rev. Lett. 119, 161101 (2017) [arXiv:1710.05832 [gr-qc]].

[3] C. Cutler and E. E. Flanagan, Phys. Rev. D 49, 2658-2697 (1994) [arXiv:gr-qc/9402014 [gr-qc]].

[4] E. Berti, V. Cardoso and C. M. Will, Phys. Rev. D 73, 064030 (2006) [gr-qc/0512160].

[5] P. Pani, V. Cardoso and L. Gualtieri, Phys. Rev. D 83, 104048 (2011) [arXiv:1104.1183 [gr-qc]].

[6] K. Yagi, L. C. Stein, N. Yunes and T. Tanaka, Phys. Rev. D 85, 064022 (2012) [arXiv:1110.5950 [gr-qc]].

[7] K. Yagi, N. Yunes and T. Tanaka, Phys. Rev. Lett. 109, 251105 (2012).

[8] L. Blanchet, Living Rev. Rel. 17, 2 (2014) [arXiv:1310.1528 [gr-qc]].

[9] E. Berti et al., Class. Quant. Grav. 32, 243001 (2015) [arXiv:1501.07274 [gr-qc]].

[10] L. Barack et al., Class. Quant. Grav. 36, 143001 (2019) [arXiv:1806.05195 [gr-qc]].

[11] E. E. Flanagan and T. Hinderer, Phys. Rev. D 77, 021502 (2008) [arXiv:0709.1915 [astro-ph]].

[12] T. Hinderer, Astrophys. J. 677, 1216-1220 (2008) [arXiv:0711.2420 [astro-ph]].

[13] T. Hinderer, B. D. Lackey, R. N. Lang and J. S. Read, Phys. Rev. D 81, 123016 (2010) [arXiv:0911.3535 [astro-ph.HE]].

[14] B. P. Abbott et al., Phys. Rev. Lett. 121, 161101 (2018) [arXiv:1805.11581 [gr-qc]].

[15] S. De, D. Finstad, J. M. Lattimer, D. A. Brown, E. Berger and C. M. Biwer, Phys. Rev. Lett. 121, 091102 (2018) [arXiv:1804.08583 [astro-ph.HE]].

[16] K. D. Kokkotas and B. G. Schmidt, Living Rev. Rel. 2, 2 (1999) [arXiv:gr-qc/9909058 [gr-qc]].

[17] H. P. Nollert, Class. Quant. Grav. 16, R159-R216 (1999).

[18] E. Berti, V. Cardoso and A. O. Starinets, Class. Quant. Grav. 26, 163001 (2009) [arXiv:0905.2975 [gr-qc]].

[19] C. M. Will, Living Rev. Rel. 9, 3 (2006) [gr-qc/0510072].

[20] E. J. Copeland, M. Sami and S. Tsujikawa, Int. J. Mod. Phys. D 15, 1753 (2006) [hep-th/0603057].

[21] A. Silvestri and M. Trodden, Rept. Prog. Phys. 72, 096901 (2009) [arXiv:0904.0024 [astro-ph.CO]]. 
[22] T. Clifton, P. G. Ferreira, A. Padilla and C. Skordis, Phys. Rept. 513, 1 (2012) [arXiv:1106.2476 [astro-ph.CO]].

[23] R. Kase and S. Tsujikawa, Int. J. Mod. Phys. D 28, 1942005 (2019) [arXiv:1809.08735 [gr-qc]].

[24] A. Joyce, B. Jain, J. Khoury and M. Trodden, Phys. Rept. 568, 1 (2015) [arXiv:1407.0059 [astro-ph.CO]].

[25] Y. Fujii and K. Maeda, "The Scalar-Tensor Theory of Gravitation", Cambridge Univ. Press (2002).

[26] G. W. Horndeski, Int. J. Theor. Phys. 10, 363-384 (1974).

[27] C. Deffayet, X. Gao, D. A. Steer and G. Zahariade, Phys. Rev. D 84, 064039 (2011) [arXiv:1103.3260 [hep-th]].

[28] T. Kobayashi, M. Yamaguchi and J. Yokoyama, Prog. Theor. Phys. 126, 511 (2011) [arXiv:1105.5723 [hep-th]].

[29] C. Charmousis, E. J. Copeland, A. Padilla and P. M. Saffin, Phys. Rev. Lett. 108, 051101 (2012) [arXiv:1106.2000 [hep-th]].

[30] J. E. Chase, Commun. Math. Phys. 19, 276 (1970).

[31] J. D. Bekenstein, Phys. Rev. Lett. 28, 452 (1972).

[32] S. W. Hawking, Commun. Math. Phys. 25, 167 (1972).

[33] J. D. Bekenstein, Phys. Rev. D 51, R6608 (1995).

[34] T. P. Sotiriou and V. Faraoni, Phys. Rev. Lett. 108, 081103 (2012) [arXiv:1109.6324 [gr-qc]].

[35] C. Brans and R. H. Dicke, Phys. Rev. 124, 925-935 (1961).

[36] T. P. Sotiriou and S. Y. Zhou, Phys. Rev. Lett. 112, 251102 (2014) [arXiv:1312.3622 [gr-qc]].

[37] T. P. Sotiriou and S. Y. Zhou, Phys. Rev. D 90, 124063 (2014) [arXiv:1408.1698 [gr-qc]].

[38] M. Rinaldi, Phys. Rev. D 86, 084048 (2012) [arXiv:1208.0103 [gr-qc]].

[39] A. Anabalon, A. Cisterna and J. Oliva, Phys. Rev. D 89, 084050 (2014) [arXiv:1312.3597 [gr-qc]].

[40] M. Minamitsuji, Phys. Rev. D 89, 064017 (2014) [arXiv:1312.3759 [gr-qc]].

[41] E. Babichev and C. Charmousis, JHEP 1408, 106 (2014) [arXiv:1312.3204 [gr-qc]].

[42] T. Kobayashi and N. Tanahashi, PTEP 2014, $073 \mathrm{E} 02$ (2014) [arXiv:1403.4364 [gr-qc]].

[43] H. Ogawa, T. Kobayashi and T. Suyama, Phys. Rev. D 93, 064078 (2016) [arXiv:1510.07400 [gr-qc]].

[44] E. Babichev, C. Charmousis and A. Lehébel, Class. Quant. Grav. 33, 154002 (2016) [arXiv:1604.06402 [gr-qc]].

[45] J. Khoury, M. Trodden and S. S. C. Wong, JCAP 11, 044 (2020) [arXiv:2007.01320 [astro-ph.CO]].

[46] T. Damour and G. Esposito-Farese, Phys. Rev. Lett. 70, 2220 (1993).

[47] T. Damour and G. Esposito-Farese, Phys. Rev. D 54, 1474 (1996) [gr-qc/9602056].

[48] A. Cooney, S. DeDeo and D. Psaltis, Phys. Rev. D 82, 064033 (2010) [arXiv:0910.5480 [astro-ph.HE]].

[49] A. S. Arapoglu, C. Deliduman and K. Y. Eksi, JCAP 1107, 020 (2011) [arXiv:1003.3179 [gr-qc]].

[50] M. Orellana, F. Garcia, F. A. Teppa Pannia and G. E. Romero, Gen. Rel. Grav. 45, 771 (2013) [arXiv:1301.5189 [astroph.CO]].

[51] A. V. Astashenok, S. Capozziello and S. D. Odintsov, JCAP 1312, 040 (2013) [arXiv:1309.1978 [gr-qc]].

[52] A. Ganguly, R. Gannouji, R. Goswami and S. Ray, Phys. Rev. D 89, 064019 (2014) [arXiv:1309.3279 [gr-qc]].

[53] S. S. Yazadjiev, D. D. Doneva, K. D. Kokkotas and K. V. Staykov, JCAP 1406, 003 (2014) [arXiv:1402.4469 [gr-qc]].

[54] M. Aparicio Resco, A. de la Cruz-Dombriz, F. J. Llanes Estrada and V. Zapatero Castrillo, Phys. Dark Univ. 13, 147 (2016) [arXiv:1602.03880 [gr-qc]].

[55] R. Kase and S. Tsujikawa, JCAP 09, 054 (2019) [arXiv:1906.08954 [gr-qc]].

[56] D. D. Doneva and S. S. Yazadjiev, Phys. Rev. Lett. 120, 131103 (2018) [arXiv:1711.01187 [gr-qc]].

[57] H. O. Silva, J. Sakstein, L. Gualtieri, T. P. Sotiriou and E. Berti, Phys. Rev. Lett. 120, 131104 (2018) [arXiv:1711.02080 $[\mathrm{gr}-\mathrm{qc}]]$.

[58] G. Antoniou, A. Bakopoulos and P. Kanti, Phys. Rev. Lett. 120, 131102 (2018) [arXiv:1711.03390 [hep-th]].

[59] G. Antoniou, A. Bakopoulos and P. Kanti, Phys. Rev. D 97, 084037 (2018) [arXiv:1711.07431 [hep-th]].

[60] M. Minamitsuji and T. Ikeda, Phys. Rev. D 99, 044017 (2019) [arXiv:1812.03551 [gr-qc]].

[61] P. V. P. Cunha, C. A. R. Herdeiro and E. Radu, Phys. Rev. Lett. 123, 011101 (2019) [arXiv:1904.09997 [gr-qc]].

[62] A. V. Frolov, Phys. Rev. Lett. 101, 061103 (2008) [arXiv:0803.2500 [astro-ph]].

[63] T. Kobayashi and K. i. Maeda, Phys. Rev. D 78, 064019 (2008) [arXiv:0807.2503 [astro-ph]].

[64] S. Tsujikawa, T. Tamaki and R. Tavakol, JCAP 05, 020 (2009) [arXiv:0901.3226 [gr-qc]].

[65] A. Upadhye and W. Hu, Phys. Rev. D 80, 064002 (2009) [arXiv:0905.4055 [astro-ph.CO]].

[66] A. A. Starobinsky, Phys. Lett. B 91, 99 (1980).

[67] A. Dohi, R. Kase, R. Kimura, K. Yamamoto and M. a. Hashimoto, PTEP 2021 (2021) no.9, 093E01 [arXiv:2003.12571 $[\mathrm{gr}-\mathrm{qc}]]$.

[68] A. Cisterna, T. Delsate and M. Rinaldi, Phys. Rev. D 92, 044050 (2015) [arXiv:1504.05189 [gr-qc]].

[69] A. Cisterna, M. Cruz, T. Delsate and J. Saavedra, Phys. Rev. D 92, 104018 (2015) [arXiv:1508.06413 [gr-qc]].

[70] A. Maselli, H. O. Silva, M. Minamitsuji and E. Berti, Phys. Rev. D 93, 124056 (2016) [arXiv:1603.04876 [gr-qc]].

[71] T. Kobayashi, H. Motohashi and T. Suyama, Phys. Rev. D 85, 084025 (2012) [erratum: Phys. Rev. D 96, 109903 (2017)] [arXiv:1202.4893 [gr-qc]].

[72] T. Kobayashi, H. Motohashi and T. Suyama, Phys. Rev. D 89, 084042 (2014) [arXiv:1402.6740 [gr-qc]].

[73] O. J. Tattersall and P. G. Ferreira, Phys. Rev. D 97, 104047 (2018) [arXiv:1804.08950 [gr-qc]].

[74] G. Franciolini, L. Hui, R. Penco, L. Santoni and E. Trincherini, JHEP 02, 127 (2019) [arXiv:1810.07706 [hep-th]].

[75] K. Glampedakis and H. O. Silva, Phys. Rev. D 100, 044040 (2019) [arXiv:1906.05455 [gr-qc]].

[76] O. J. Tattersall, Class. Quant. Grav. 37, 115007 (2020) [arXiv:1911.07593 [gr-qc]].

[77] K. Tomikawa and T. Kobayashi, Phys. Rev. D 103, 084041 (2021) [arXiv:2101.03790 [gr-qc]].

[78] D. Langlois, K. Noui and H. Roussille, Phys. Rev. D 104 (2021) no.12, 124044 [arXiv:2103.14750 [gr-qc]].

[79] R. Kase, R. Kimura, S. Sato and S. Tsujikawa, Phys. Rev. D 102, 084037 (2020) [arXiv:2007.09864 [gr-qc]].

[80] R. Kase and S. Tsujikawa, JCAP 01, 008 (2021) [arXiv:2008.13350 [gr-qc]]. 
[81] A. Goldstein et al., Astrophys. J. 848, L14 (2017) [arXiv:1710.05446 [astro-ph.HE]].

[82] B. F. Schutz and R. Sorkin, Annals Phys. 107, 1 (1977).

[83] J. D. Brown, Class. Quant. Grav. 10, 1579 (1993) [gr-qc/9304026].

[84] A. De Felice, J. M. Gerard and T. Suyama, Phys. Rev. D 81, 063527 (2010) [arXiv:0908.3439 [gr-qc]].

[85] A. De Felice, L. Heisenberg, R. Kase, S. Mukohyama, S. Tsujikawa and Y. l. Zhang, JCAP 06, 048 (2016) [arXiv:1603.05806 [gr-qc]].

[86] L. Amendola and S. Tsujikawa, JCAP 06, 020 (2020) [arXiv:2003.02686 [gr-qc]].

[87] M. Minamitsuji and S. Tsujikawa, Phys. Lett. B 820, 136509 (2021) [arXiv:2105.14661 [gr-qc]].

[88] T. Regge and J. A. Wheeler, Phys. Rev. 108, 1063-1069 (1957).

[89] F. J. Zerilli, Phys. Rev. Lett. 24, 737-738 (1970).

[90] A. De Felice, T. Suyama and T. Tanaka, Phys. Rev. D 83, 104035 (2011) [arXiv:1102.1521 [gr-qc]].

[91] H. Motohashi and T. Suyama, Phys. Rev. D 84, 084041 (2011) [arXiv:1107.3705 [gr-qc]].

[92] R. Kase, L. Á. Gergely and S. Tsujikawa, Phys. Rev. D 90, 124019 (2014) [arXiv:1406.2402 [hep-th]].

[93] C. de Rham, J. Francfort and J. Zhang, Phys. Rev. D 102, 024079 (2020) [arXiv:2005.13923 [hep-th]].

[94] R. Kase, M. Minamitsuji, S. Tsujikawa and Y. L. Zhang, JCAP 02, 048 (2018) [arXiv:1801.01787 [gr-qc]].

[95] C. T. Cunningham, R. H. Price and V. Moncrief, Astrophys. J. 224, 643 (1978).

[96] R. Kase, M. Minamitsuji and S. Tsujikawa, Phys. Rev. D 102, 024067 (2020) [arXiv:2001.10701 [gr-qc]].

[97] S. Tsujikawa, C. Zhang, X. Zhao and A. Wang, Phys. Rev. D 104, 064024 (2021) [arXiv:2107.08061 [gr-qc]].

[98] J. L. Blázquez-Salcedo and K. Eickhoff, Phys. Rev. D 97, 104002 (2018) [arXiv:1803.01655 [gr-qc]].

[99] Z. Altaha Motahar, J. L. Blázquez-Salcedo, D. D. Doneva, J. Kunz and S. S. Yazadjiev, Phys. Rev. D 99, 104006 (2019) [arXiv:1902.01277 [gr-qc]]. 